This item was submitted to Loughborough's Research Repository by the author.

Items in Figshare are protected by copyright, with all rights reserved, unless otherwise indicated.

\title{
Stochastic heat and Burgers equations and their singularities. I. Geometrical
} properties

PLEASE CITE THE PUBLISHED VERSION

LICENCE

CC BY-NC-ND 4.0

\section{REPOSITORY RECORD}

Davies, lan M., Aubrey Truman, and Huaizhong Zhao. 2019. "Stochastic Heat and Burgers Equations and Their Singularities. I. Geometrical Properties”. figshare. https://hdl.handle.net/2134/711. 


\title{
Stochastic Heat and Burgers Equations and Their Singularities I - Geometrical Properties Dedicated to V. I. Arnol'd
}

\author{
Ian M Davies, Aubrey Truman, Huaizhong Zhao ${ }^{\dagger}$
}

November 21, 2001

\begin{abstract}
Arnol'd and Thom's beautiful classification of caustics (shockwaves) for Burgers equation suggests a similar one for the wavefronts of the corresponding Heat equation. We give here a general theorem for Hamiltonian systems characterising how the level surfaces of Hamilton's principal function (wavefronts) meet the caustic surface in both the deterministic and stochastic cases. We further show how these results can be applied to the stochastic Burgers equation by using earlier results of Truman and Zhao.

The generic example of a caustic, appearing in the two dimensional case, is the semicubical parabolic cusp with the corresponding zero level surface being a combination of a generalised hypocycloid and a line pair. We refer to these as the Cusp and Tricorn. The analogous Butterfly caustic, in the three dimensional case, has a cusped zero level surface, the Fish, which meets the Butterfly caustic in three cusped curves and touches it along a straight line. Our results explain in terms of classical mechanics the properties of the caustic and wavefront for these two archetypal examples and characterise the caustic-wavefront intersection for the general stochastic case.

We discuss the application of these results to turbulence for the Burgers velocity field.

A.M.S. Classification: Primary 60H15, 60H30, 35Q53, Secondary 76M $35,35 \mathrm{R} 60,58 \mathrm{~K} 45$
\end{abstract}

${ }^{*}$ Department of Mathematics, University of Wales Swansea, Singleton Park, Swansea, SA2 8PP, Wales, UK. Email: I.M.Davies@swansea.ac.uk, A.Truman@swansea.ac.uk

${ }^{\dagger}$ Department of Mathematical Sciences, Loughborough University, Loughborough, Leicestershire, LE11 3TU, England, UK. Email: H.Zhao@lboro.ac.uk 


\section{Introduction}

Stochastic Burgers equations have attracted a considerable amount of attention in recent years, e.g. [1], [4], [8], [13], [14], [22], [23], [24], [25], [26], [30], [33], [35], [36]. See also [2], [5], [6], [7], [20], [28] for related works. They have been used to give models of turbulence (see especially [13]) and to model the large scale structure of the universe [38]. Here we shall be interested in what has come to be called Burgulence.

Consider the stochastic viscous Burgers equation for the velocity field $v^{\mu}=v^{\mu}(x, t), x \in \mathbb{R}^{d}, t>0$,

$$
\frac{\partial v^{\mu}}{\partial t}+\left(v^{\mu} \cdot \nabla\right) v^{\mu}=\frac{\mu^{2}}{2} \Delta v^{\mu}-\nabla c(x)-\epsilon \nabla k(x, t) \dot{W}_{t}
$$

with initial velocity $v^{\mu}(x, 0)=\nabla S_{0}(x)$ where $\mu^{2}$ is the coefficient of viscosity. Here $c$ and $k$ are $C^{2}$ functions and $W_{t}$ is a Wiener process on the probability space $\{\Omega, \mathcal{F}, P\}$. We shall be interested in the 'blow-up' of $v^{0}(x, t)$ where

$$
v^{0}(x, t)=\lim _{\mu \rightarrow 0} v^{\mu}(x, t)
$$

i.e. the advent of discontinuities in $v^{0}$.

The corresponding heat equation for $u^{\mu}=u^{\mu}(x, t)$ is the Stratonovich equation

$$
\begin{aligned}
\frac{\partial u^{\mu}}{\partial t} & =\frac{\mu^{2}}{2} \Delta u^{\mu}+\frac{1}{\mu^{2}} c(x) u^{\mu}+\frac{\epsilon}{\mu^{2}} k(x, t) u^{\mu} \circ \dot{W}_{t}, \\
u^{\mu}(x, 0) & =\exp \left(-S_{0}(x) / \mu^{2}\right),
\end{aligned}
$$

the connection between $u^{\mu}$ and $v^{\mu}$ being the Hopf-Cole logarithmic transformation $v^{\mu}=-\mu^{2} \nabla \ln u^{\mu}$.

Following Donsker, Freidlin et al $[19,21]$ we expect as $\mu \rightarrow 0$,

$$
-\mu^{2} \ln u^{\mu}(x, t) \rightarrow \inf _{X(0)}\left[A(X(0), x, t)+S_{0}(X(0))\right]=S(x, t),
$$

with

$$
A(X(0), x, t)=\inf _{\substack{X(s) \\ X(t)=x}} A[X]
$$

where $A[X]$ is the stochastic action

$$
A(X(0), x, t)=\frac{1}{2} \int_{0}^{t} \dot{X}^{2}(s) d s-\int_{0}^{t} c(X(s)) d s-\epsilon \int_{0}^{t} k(X(s), s) d W_{s} .
$$


$S(x, t)$ is the solution of the stochastic Hamilton-Jacobi equation

$$
d S_{t}+\frac{|\nabla S|^{2}}{2} d t+c(x) d t+\epsilon k(x, t) d W_{t}=0, \quad S(x, 0)=S_{0}(x),
$$

and so $S(x, t)$ is Hamilton's principal function for a stochastic mechanical path. To see this observe that if $\dot{X}(s)$ is a continuous process with the integration by parts property $\left(\mathrm{ibp}^{2}\right)$ : for all (deterministic) $u \in C^{1}(0, t)$

$$
\int_{0}^{t} \dot{u}(s) \dot{X}(s) d s=u(t) \dot{X}(t)-u(0) \dot{X}(0)-\int_{0}^{t} u(s) d \dot{X}(s)
$$

setting $u(t)=0, \mathcal{A}[X]=A[X]+S_{0}(X(0))$, we easily obtain for $S_{0} \in C^{1}$, $c \in C^{1}, k \in C^{1,0}$

$$
\begin{aligned}
& -\left.\frac{d}{d \eta}\right|_{\eta=0} \mathcal{A}[X+\eta u] \\
& =\int_{0}^{t} u(s)\left[d \dot{X}(s)+\nabla c(X(s)) d s+\epsilon \nabla k(X(s), s) d W_{s}\right] \\
& \quad+u(0)\left(\dot{X}(0)-\nabla S_{0}(X(0))\right) .
\end{aligned}
$$

Hence necessary conditions for the extremizer are, for $s \in[0, t]$,

$$
d \dot{X}(s)+\nabla c(X(s)) d s+\epsilon \nabla k(X(s), s) d W_{s}=0, \quad \dot{X}(0)=\nabla S_{0}(X(0)) .
$$

From Equation (1.3), as $\mu \rightarrow 0$, we expect that $u^{\mu}$ switches from being exponentially large to exponentially small across the level surface

$$
S(x, t)=0 . \quad(\text { zero level surface })
$$

If we demand that $X(t)=x$, for fixed $t$ and $x, X(s)$ satisfying Equation (1.7) may not be unique. Hence, we expect that shockwaves for $v$ arise from precaustics (in $\left(x_{0}, t\right)$ variables) when infinitely many of these classical mechanical paths from $x_{0}$ and a neighbourhood focus in a set of zero volume centred on $X(t)$. The condition for paths starting from $x_{0}$ focusing at a point $X$ at time $t$ is

$$
\operatorname{Det}\left(\frac{\partial X(t)}{\partial x_{0}}\right)=0 . \quad \text { (precaustic) }
$$

Define the random map $\Phi_{s}: \mathbb{R}^{d} \rightarrow \mathbb{R}^{d}$ corresponding to the classical flow by the second order stochastic differential equation

$$
d_{s} \dot{\Phi}_{s}=-\nabla c\left(\Phi_{s}\right) d s-\nabla k\left(\Phi_{s}, s\right) d W_{s}
$$


with $\Phi_{0}=I$ and $\dot{\Phi}_{0}=\nabla S_{0}$ so that $X(s)=\Phi_{s} \Phi_{t}^{-1} x$, where we accept that $x_{0}(x, t)=\Phi_{t}^{-1} x$ is not necessarily unique. Given some regularity, the global inverse function theorem gives a caustic time $T(\omega)$ such that, for $s<T(\omega)$, $\Phi_{s}$ is a random diffeomorphism [37]. Therefore, as we shall see,

$$
v^{0}(x, t)=\dot{\Phi}_{t} \Phi_{t}^{-1} x=\nabla S(x, t)
$$

is a formal solution of Burgers equation with $\mu=0$, which is well defined up to the caustic time because $x_{0}(x, t)$ is unique.

After the caustic time, for polynomial $S_{0}, x_{0}(x, t)$ will usually have finite multiplicity. As long as the minimising $x_{0}(x, t)$ is unique, Equation (1.10) can be assumed to be true beyond the caustic time if we work with that part of the level surface of Hamilton's principal function corresponding to the minimising $x_{0}(x, t)$. This is equivalent to taking the minimum entropy solution of Burgers equation [29]. As expected the main contribution to $\lim _{\mu \rightarrow 0} v^{\mu}$ will come from the minimising $x_{0}(x, t)$ which we shall assume is unique. When there are a finite number $(>1)$ of minimising $x_{0}$ 's the limiting solution, $v^{0}$, is more complicated [9], but can still be written down.

We expect the non-uniqueness of $x_{0}(x, t)$ to be associated with the appearance of discontinuities in $v^{0}(x, t)$ and $u^{0}(x, t)$. An obvious way for these to arise is if (as above) infinitely many paths $X(s)$ focus in zero volume centred at $x$. Eliminating $x_{0}$ by using $x=\Phi_{t}\left(x_{0}\right)$ gives the equation of the caustic $C_{t}$

$$
\left.\operatorname{Det}\left(\frac{\partial X(t)}{\partial x_{0}}\right)\right|_{x_{0}=\Phi_{t}^{-1} x}=0 . \quad \text { (caustic) }
$$

The precaustic $\Phi_{t}^{-1} C_{t}$ has defining equation (1.8). Starting with the level surfaces of Hamilton's principal function $H_{t}$ one can define the prelevel surfaces $\Phi_{t}^{-1} H_{t}$ in a similar way by setting $x=\Phi_{t}\left(x_{0}\right)$. The prelevel surfaces and the precaustic are just the preimages of the level surfaces and the caustic under the classical flow map $\Phi_{t}$. The wavefront is the zero level set of the minimising Hamilton function which as we shall see is part of $H_{t}$. For a non-degenerate critical point, when the multiplicity of $x_{0}(x, t)$, $n=n(x, t)$, is finite so that $\Phi_{t}^{-1}\{x\}=\left\{x_{0}^{1}(x, t), x_{0}^{2}(x, t), \ldots, x_{0}^{n}(x, t)\right\}$, from $[9,10]$ and $[16,17,18]$ we can deduce that

$$
u^{\mu}(x, t) \sim \sum_{i=1}^{n} \theta_{i} \exp \left\{-S_{0}^{i}(x, t) / \mu^{2}\right\},
$$

where

$$
S_{0}^{i}(x, t)=S_{0}\left(x_{0}^{i}(x, t)\right)+A\left(x_{0}^{i}(x, t), x, t\right),
$$


for $i=1,2, \ldots, n$ and $\theta_{i}$ is an asymptotic series in $\mu^{2}$ whose detailed structure is discussed below. Here $H_{t}=\left\{x: S_{0}^{i}(x, t)=0\right.$, some $\left.i\right\}$ so it includes the wavefront where $u^{0}$ switches smoothly from being exponentially large to exponentially small. Needless to say the dominant term in Equation (1.12) comes from the minimising $x_{0}(x, t)$ so that

$$
S(x, t)=\min _{i=1,2, \ldots, n} S_{0}^{i}(x, t)
$$

in line with the results of Freidlin et al. Of course $u^{0}(x, t)$ can switch discontinuously from being exponentially large to exponentially small as we cross parts of the caustic since the minimising $S_{0}^{i}$ can disappear. 
Example 1.1. The paradigm for $c=k \equiv 0$ is when $S_{0}(x, y)=x^{2} y / 2$ in the two-dimensional case. This gives us the generic cusp catastrophe.

Figure 1. Cusp and Tricorn

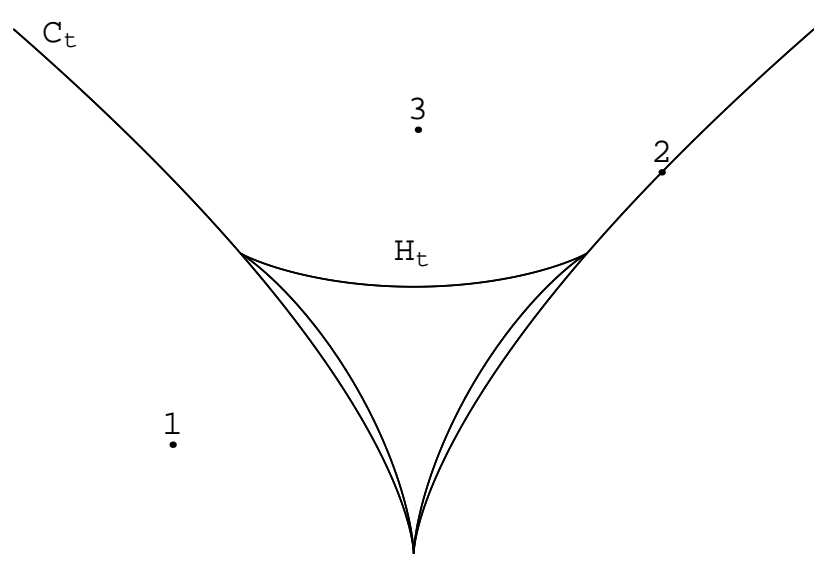

The Caustic (Shockwave), $C_{t}$, with Equation (4.2) is the classical semicubical parabola (Cusp). This gives the surface of discontinuity of $v^{0}(x, t)$ with $v^{0}(x, 0)=\nabla S_{0}=\left(x y, x^{2} / 2\right)$. The corresponding zero level surface of Hamilton's principal function, $H_{t}$, is a generalized hypocycloid (Tricorn) with Equation (4.1) and line pair $x^{2}=0$. Only the top part of this Tricorn forms the wavefront. The other two curves come from spurious zeros of $S_{0}^{i}$ where $S_{0}^{i}$ is not minimising. This top part of the Tricorn defines the region where $u^{\mu}$ switches from being exponentially large to exponentially small. We suppress the line pair in the above figure to highlight how the Caustic splits $\mathbb{R}^{2}$. The important result here is that $u^{0}$ has a sharp discontinuity on the Caustic itself. Note that within the Cusp there are three pre-images for each point, on the Cusp there are two pre-images for each point and outwith the Cusp there is only one pre-image for each point.

If we fix the time $t$ and let the point a be inside the semicubical parabolic Cusp so that the multiplicity of the $x_{0}$ 's is three, there are three level surfaces $S_{0}^{i}(x, t)=c$ one for each $x_{0}^{i}(a, t), i=1,2,3$, only one of which corresponds to the minimiser. The prelevel surfaces and their corresponding $x_{0}$ 's are shown below with the Cusp included for reference. 
Figure 2. 3 Prelevel surfaces - positive

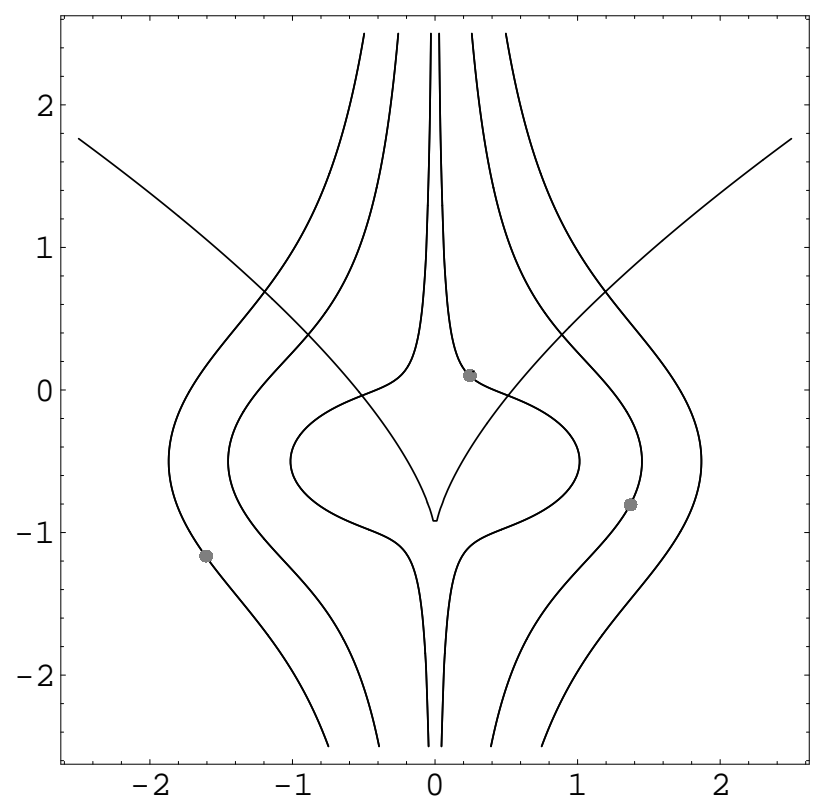

Figure 3. 3 Prelevel surfaces - two negative and one positive

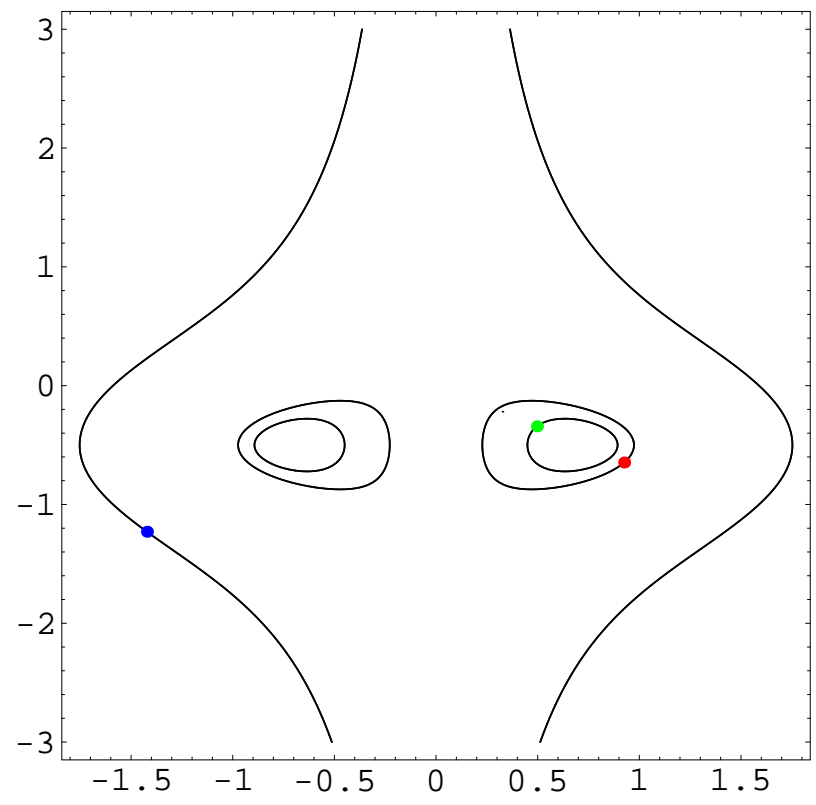


We now display all the level surfaces passing through the point a in relation to the Cusp.

Figure 4. 3 Level surfaces - positive

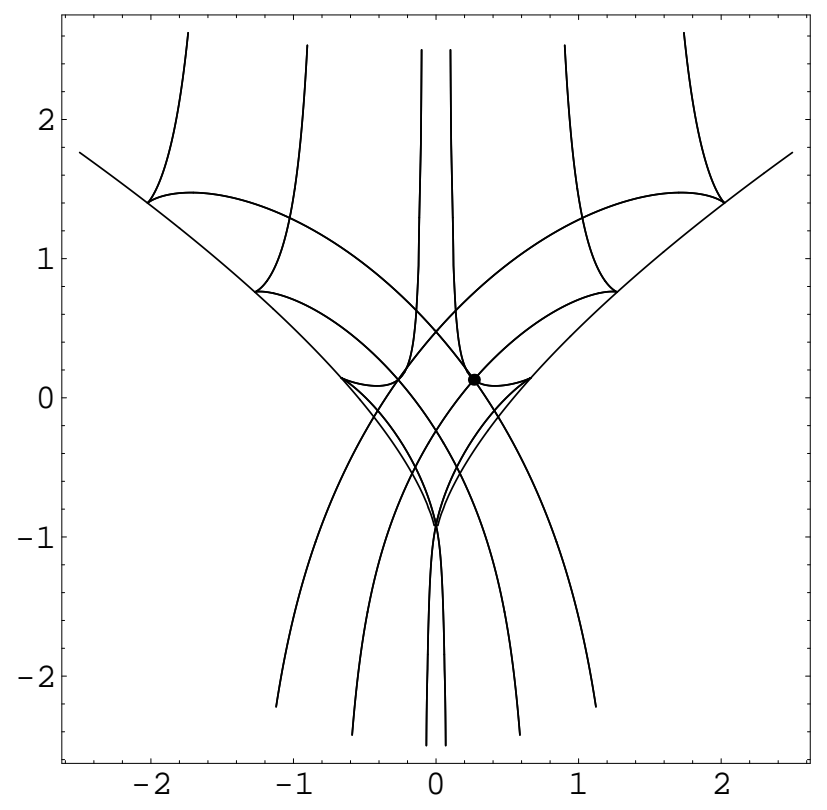

Figure 5. 3 Level surfaces - two negative and one positive

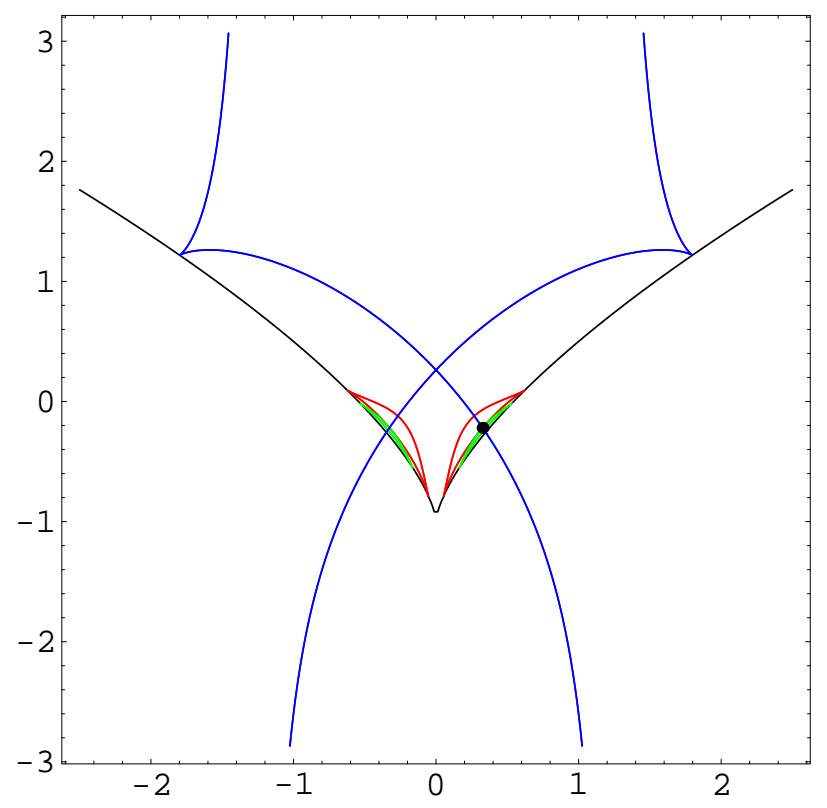


In the case when the point $a$ is on the Cusp there are only two preimage points and correspondingly only two level surfaces passing through a, one of which has a cusp at a.

Figure 6. 2 Level surfaces

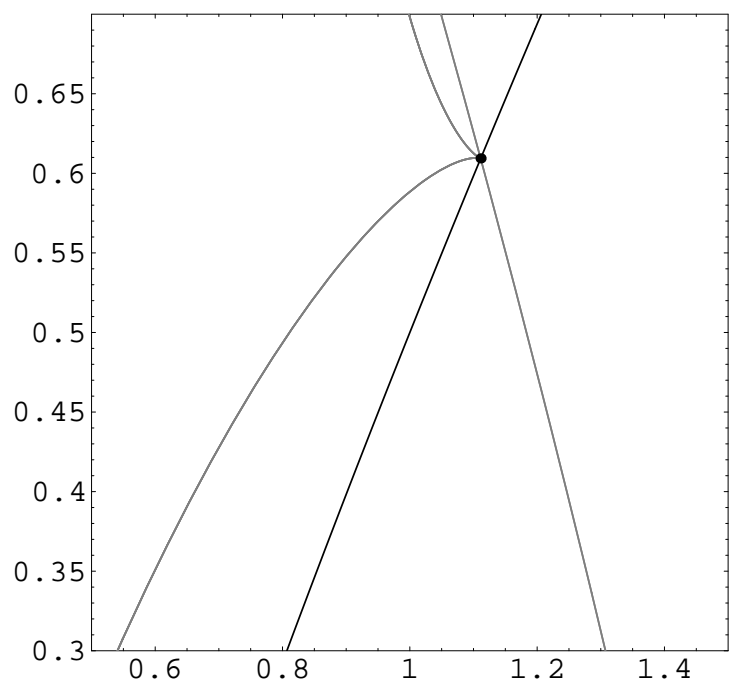

The final case is where the point a lies outwith the Cusp and there is only one level surface.

Figure 7. 1 Level surface

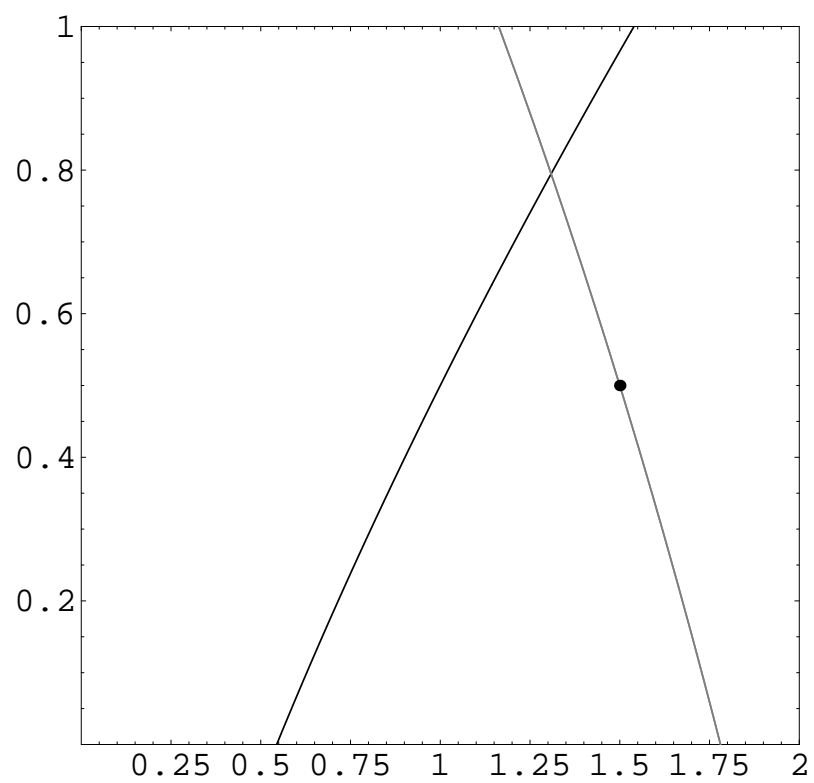


In the following two figures we see how the cusped level surfaces coalesce as the point a approaches the Caustic. The first of these corresponds to the two cusped level surfaces having positive $S_{0}$ and the second corresponds to the two cusped level surfaces having negative $S_{0}$. In both cases the appearance of the cusp means that the corresponding $S_{0}^{i}$ cannot be continued across the Caustic. This is what makes $u^{0}$ discontinuous across the Cusp caustic, because the cusped part of the level surface corresponds to the minimiser $x_{0}(x, t)$.

Figure 8. Coalescing level surfaces - positive $S_{0}$

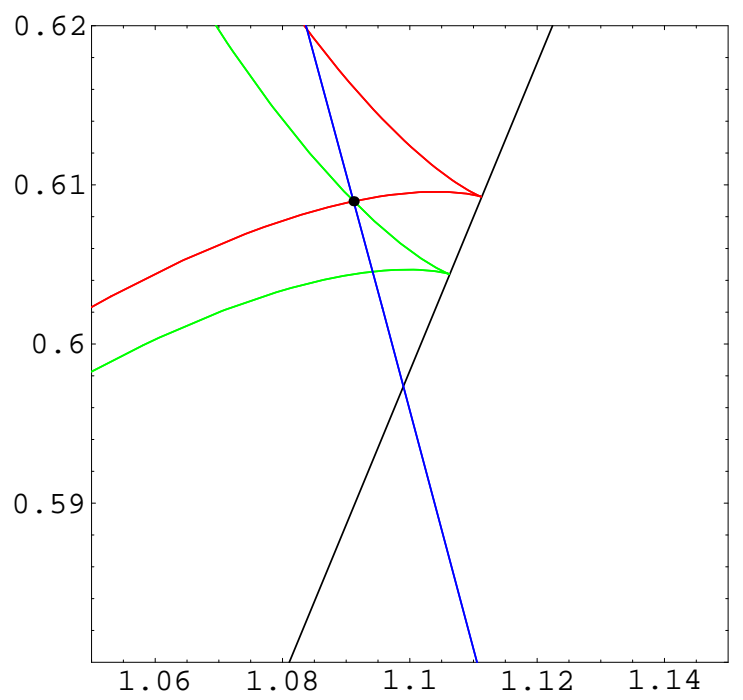

Figure 9. Coalescing level surfaces - negative $S_{0}$

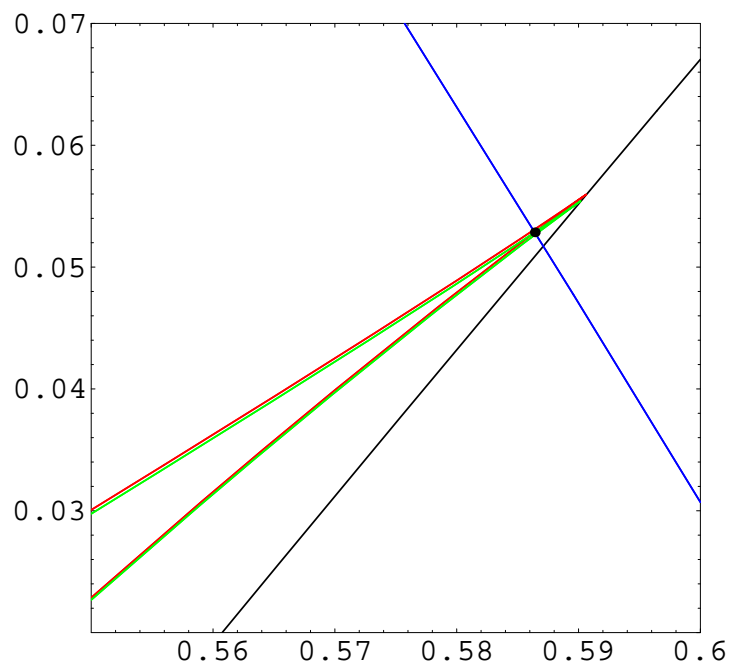


In the above example we see that the multiplicity of $x_{0}(x, t), n$, changes by a multiple of two as we cross the caustic surface. We shall see that this is associated with level surfaces of Hamilton's principal function having cusps on the caustic, caused by different $x_{0}(x, t)$ 's coalescing. Moreover the cusp in the level surface (if it corresponds to the minimising $x_{0}$ ) means that we have to take a different minimiser $S_{0}^{i}$ on different sides of the caustic. This makes $u^{0}$ discontinuous. Thus, it is important to know when the point of intersection of a level surface and caustic is a genuine cusp, generalised cusp or a crossover point.

In more than one dimension for $S_{0} \in C^{2}$ there is a robust geometrical relationship between level surfaces and caustics even in the presence of noise. This is because, as we shall prove, the tangent plane to the level surface $H_{t}$ at a point $x$ on the caustic $C_{t}$ is spanned by the image of the tangent plane to the prelevel surface, where this prelevel surface tangent plane necessarily contains the kernel of the derivative of the $\Phi_{t}$ map if $x \in \Phi_{t}\left(\Phi_{t}^{-1} C_{t} \cap \Phi_{t}^{-1} H_{t}\right)$. In understanding Burgers turbulence this switches attention from $C_{t}$ and $H_{t}$ to their unfoldings $\Phi_{t}^{-1} C_{t}$ and $\Phi_{t}^{-1} H_{t}$. In two dimensions, at a point on the precaustic it follows that this kernel spans the tangent plane to the prelevel surface. In three dimensions the dimension of the tangent plane to the level surface drops from two to one as we approach points on the caustic in $\Phi_{t}\left[\Phi_{t}^{-1} H_{t} \cap \Phi_{t}^{-1} C_{t}\right]$ which is essentially the cusped part of the level surface, $\operatorname{Cusp}\left(H_{t}\right)$. This manifests itself in the tangent plane to the level surface on the caustic folding back on itself - the fold being the one dimensional tangent plane at the point of intersection. In two dimensions this is quite elementary to prove, but in three dimensions it depends on a careful analysis of the geometry of the level surface and its pre-image. Our main theorem (in Section 5) concerning this geometric relationship for the above stochastic Hamiltonian system, given some mild restrictions, is for fixed $t$ :

Theorem. Any point $x$ on the level surface $H_{t}, x=\Phi_{t}\left(x_{0}\right)$ with $x_{0}=\Phi_{t}^{-1}(x)$ on the prelevel surface, can only be a generalised cusp of a curve on $H_{t}$ if $x_{0}$ is a generalised cusp of the precurve on the prelevel surface or if $x_{0} \in \Phi_{t}^{-1} C_{t}$ the precaustic. In three dimensions the planar cross section $y=$ constant (with normal $e_{y}$ ) of the level surface $H_{t}$ through a point $x$ where it meets $C_{t}$, the caustic surface, will have a genuine cusp at $x$ if $x \in \operatorname{Cusp}\left(H_{t}\right)$ and there is a non-zero solution $\delta x$ of Equations $\left(C_{1}\right),\left(C_{2}\right)$ and $\left(C_{3}\right)$. The direction of the axis of the cusp will be $\widehat{\delta x}$.

This result explains the bizarre pictures of the Fish and the Butterfly (Figures 19, 20, 21, 22) in Section 4 and similar phenomena. In Section 2 we recapitulate our main results for Burgers equation. This can be skipped if the reader is primarily interested in the geometrical result. An outline proof 
of our main geometrical result is given in Section 3 where the free case is considered in detail and proved. Section 4 contains simple archetypal examples in both two and three dimensions. Section 5 treats the general stochastic case. Sections 3, 4 and 5 give a brief discussion of the ramifications of our results for Burgers turbulence. In particular we explain how our results give a simple model for the intermittence of turbulence for the stochastic Burgers equation at the end of Section 5. 


\section{Solutions of inviscid Burgers and Heat Equa- tions and asymptotic series $\theta$}

Here let $v^{\mu}=v^{\mu}(x, t)$ satisfy for $t>0, x \in \mathbb{R}^{d}$,

$$
d v^{\mu}+\left(v^{\mu} \cdot \nabla\right) v^{\mu} d t+\nabla c d t+\epsilon \nabla k d W_{t}=\frac{\mu^{2}}{2} \Delta v^{\mu} d t,
$$

with $v^{\mu}(x, 0)=\nabla S_{0}(x)$, where $c=c(x), k=k(x, t)$.

Since the convected derivative

$$
\frac{D}{D t}=\frac{\partial}{\partial t}+v \cdot \nabla
$$

the above Burgers equation describes a fluid where particles pass through each other with no interaction, the particles being subject to a force $(-\nabla c(x)-$ $\epsilon \nabla k(x, t) \dot{W}_{t}$ ) with $\dot{W}_{t}$ being white noise. (In fact infinitely many pass through each other at points on the caustic and to this extent our continuation of the solution may be considered unphysical. Nevertheless Burgers equation has been used successfully in modelling the large scale structure of the universe $[38,15]$ and in studying related equations.)

Let $v^{\mu}=\nabla S^{\mu}$. Then $S^{\mu}$ satisfies the stochastic Hamilton-Jacobi equation

$$
d S^{\mu}+\frac{1}{2}\left|\nabla S^{\mu}\right|^{2} d t+c d t+\epsilon k d W_{t}=\frac{1}{2} \mu^{2} \Delta S^{\mu} d t .
$$

Formally, if $S^{\mu} \sim \sum_{j=0}^{\infty} \mu^{2 j} S_{j}$, we obtain from the last equation the stochastic Hamilton-Jacobi continuity equations

$$
\frac{\partial S_{j}}{\partial t}+\frac{1}{2} \sum_{\substack{i_{1}, i_{2} \geq 0 \\ i_{1}+i_{2}=j}} \nabla S_{i_{1}} \cdot \nabla S_{i_{2}}=\frac{1}{2} \Delta S_{j-1},
$$

for $j=0,1,2, \ldots$, with the convention $\frac{1}{2} \Delta S_{-1}=-c-\epsilon k \dot{W}_{t}, \dot{W}_{t}$ being white noise.

The corresponding stochastic mechanical flow $\Phi_{s}$ satisfies

$$
d \dot{\Phi}_{s}=-\nabla c\left(\Phi_{s}\right) d s-\epsilon \nabla k\left(\Phi_{s}, s\right) d W_{s},
$$

with $\Phi_{0}(x)=x, \dot{\Phi}_{0}(x)=\nabla S(x, 0)=\nabla S_{0}(x)$. Now assume $\Phi_{s}(\omega): \mathbb{R}^{d} \rightarrow \mathbb{R}^{d}$ is a diffeomorphism for $0 \leq s<T(\omega)$. Define

$$
\tilde{S}_{0}(y, t)=\frac{1}{2} \int_{0}^{t}\left|\dot{\Phi}_{s}(y)\right|^{2} d s+S(y, 0)-\int_{0}^{t} c\left(\Phi_{s} y\right) d s-\epsilon \int_{0}^{t} k\left(\Phi_{s} y, s\right) d W_{s}
$$

and

$$
S_{0}(x, t)=\tilde{S}_{0}\left(\Phi_{t}^{-1} x, t\right)
$$

The main result of stochastic Hamilton-Jacobi theory $[35,34]$ is : 
Proposition 2.1. Assume $c \in C^{2}\left(\mathbb{R}^{d}\right), k \in C^{2}\left(\mathbb{R}^{d} \times \mathbb{R}\right)$ and $S_{0}(\cdot, 0) \in C^{2}\left(\mathbb{R}^{d}\right)$. Then

(i) For a.e. $\omega \in \Omega, 0 \leq t<T(\omega)$,

$$
\dot{\Phi}_{t}=\nabla S_{0}\left(\Phi_{t}, t\right)
$$

and $S_{0}$ satisfies the stochastic Hamilton-Jacobi equation

$$
d S_{0}(x, t)+\frac{1}{2}\left|\nabla S_{0}(x, t)\right|^{2} d t+c(x) d t+\epsilon k(x, t) d W_{t}=0 .
$$

(ii) Define $\rho(x, t)=\left|\operatorname{Det}\left(\frac{\partial}{\partial x} \Phi_{t}^{-1}(x)\right)\right|$. Then for a.e. $\omega \in \Omega$, any $x \in \mathbb{R}^{d}$, $0 \leq t<T(\omega), \rho$ satisfies the continuity equation

$$
\frac{\partial \rho}{\partial t}+\nabla \cdot\left(\rho \nabla S_{0}\right)=0 .
$$

Suppose now that $T_{0}: \mathbb{R}^{d} \rightarrow \mathbb{R}$ is a smooth positive function whose physical role is explained later. (Eventually we will set $T_{0} \equiv 1$ for the applications discussed here.) Define

$$
T_{0}(y, t)=T_{0}(y)
$$

and for $j=1,2, \ldots$,

$$
T_{j}(y, t)=\left.\int_{0}^{t} \rho^{-\frac{1}{2}}(\cdot, s) \Delta \cdot\left(\rho^{\frac{1}{2}}(\cdot, s) T_{j-1}\left(\Phi_{s}^{-1} \cdot, s\right)\right)\right|_{\Phi_{s} y} d s .
$$

Then for $j=0,1,2, \ldots$, define

$$
\psi_{j}(x, t)=T_{j}\left(\Phi_{t}^{-1} x, t\right) \rho^{\frac{1}{2}}(x, t) .
$$

Then we have:

\section{Lemma 2.1 .}

$$
\frac{\partial \psi_{j}}{\partial t}+\nabla \psi_{j} \cdot \nabla S_{0}=-\frac{1}{2} \psi_{j} \Delta S_{0}+\Delta \psi_{j-1}
$$

for $j=0,1,2, \ldots$, with the convention $\psi_{-1} \equiv 0$.

We have now assembled the main tools we need. We now explain how to solve the stochastic Hamilton-Jacobi continuity equations.

Proposition 2.2. Assume that $c \in C^{2}\left(\mathbb{R}^{d}\right), k \in C^{2}\left(\mathbb{R}^{d} \times \mathbb{R}\right), S_{0} \in C^{2}\left(\mathbb{R}^{d}\right)$ and $\Phi_{t}$ satisfies a no caustic condition. Then for a.e. $\omega \in \Omega$ solutions of the Hamilton-Jacobi continuity equations

$$
\frac{\partial S_{j}}{\partial t}+\frac{1}{2} \sum_{\substack{i_{1}, i_{2} \geq 0 \\ i_{1}+i_{2}=j}} \nabla S_{i_{1}} \cdot \nabla S_{i_{2}}=\frac{1}{2} \Delta S_{j-1}, \quad j \geq 0,
$$


(with the convention $\frac{1}{2} \Delta S_{-1}=-c-\epsilon k \dot{W}_{t}$ ) are given by

$$
S_{1}(x, t)=-\ln \psi_{0}(x, t)
$$

and for $j \geq 2$

$$
\begin{aligned}
S_{j}(x, t)=2^{1-j}\left(-\frac{\psi_{j-1}}{\psi_{0}}+\sum_{\substack{i_{1}, i_{2} \geq 1 \\
i_{1}+i_{2}=j-1}} \frac{\psi_{i_{1}} \psi_{i_{2}}}{2 \psi_{0}^{2}}-\right. \\
\\
\left.\quad-\sum_{\substack{i_{1}, i_{2}, i_{3} \geq 1 \\
i_{1}+i_{2}+i_{3}=j-1}} \frac{\psi_{i_{1}} \psi_{i_{2}} \psi_{i_{3}}}{3 \psi_{0}^{3}}+\cdots+\frac{(-1)^{j-1} \psi_{1}^{j-1}}{(j-1) \psi_{0}^{j-1}}\right)(x, t) .
\end{aligned}
$$

There is an important Corollary:

Corollary 2.1.

$\frac{\partial}{\partial t}\left(\sum_{j=0}^{m} \mu^{2 j} S_{j}\right)+\frac{1}{2} \sum_{j=0}^{m} \mu^{2 j}\left(\sum_{\substack{i_{1}, i_{2} \geq 0 \\ i_{1}+i_{2}=j}} \nabla S_{i_{1}} \cdot \nabla S_{i_{2}}\right)+c+k \dot{W}_{t}=\frac{\mu^{2}}{2} \Delta \sum_{j=0}^{m-1} \mu^{2 j} S_{j}$.

An all important role is played by the Nelson diffusion process $y_{s}^{\mu}$ with drift given by $-\nabla\left(\sum_{j=0}^{m} \mu^{2 j} S_{j}\left(y_{s}^{\mu}, t-s\right)\right)$

$$
\begin{aligned}
d y_{s}^{\mu} & =\mu d B(s)-\nabla \sum_{j=0}^{m} \mu^{2 j} S_{j}\left(y_{s}^{\mu}, t-s\right) d s \\
y_{0}^{\mu} & =x
\end{aligned}
$$

Consider the stochastic heat equation of Stratonovich type

$$
\begin{aligned}
d u_{t}^{\mu}(x) & =\left[\frac{\mu^{2}}{2} \Delta u_{t}^{\mu}(x)+\mu^{-2} c(x) u_{t}^{\mu}(x)\right] d t+\epsilon \mu^{-2} k(x, t) u_{t}^{\mu}(x) \circ d W_{t}, \\
u_{0}^{\mu}(x) & =T_{0}(x) \exp \left(-S_{0}(x) / \mu^{2}\right) .
\end{aligned}
$$

Proposition 2.3. For each $m \geq 0$,

$$
\begin{array}{r}
u_{t}^{\mu}(x)= \\
\exp \left\{-\mu^{-2} \sum_{j=0}^{m} \mu^{2 j} S_{j}(x, t)\right\} \mathbb{E} \exp \left\{-\frac{\mu^{2 m}}{2} \int_{0}^{t} \Delta S_{m}\left(y_{s}^{\mu}, t-s\right) d s\right. \\
\left.+\frac{1}{2} \sum_{j=m+1}^{2 m} \mu^{2(j-1)} \sum_{\substack{0 \leq i_{1}, i_{2} \leq m \\
i_{1}+i_{2}=j}} \int_{0}^{t} \nabla S_{i_{1}} \cdot \nabla S_{i_{2}}\left(y_{s}^{\mu}, t-s\right) d s\right\} .
\end{array}
$$


Note that the second factor is of the form $\left(1+O\left(\mu^{2 m}\right)\right)$ and the first factor gives the expansion up to $\mu^{2 m-2}$ for each $m$.

Our main result is:

Proposition 2.4. The solution of the viscous stochastic Burgers equation is for each $m \geq 0$

$$
\begin{aligned}
v^{\mu}(x, t)=\sum_{j=0}^{m} \mu^{2 j} v_{j}(x, t) & \\
-\mu^{2} \nabla \ln & \mathbb{E}\left\{\operatorname { e x p } \left\{-\frac{\mu^{2 m}}{2} \int_{0}^{t} \nabla \cdot v_{m}\left(y_{s}^{\mu}, t-s\right) d s\right.\right. \\
& \left.\left.+\frac{1}{2} \sum_{j=m+1}^{2 m} \mu^{2(j-1)} \sum_{\substack{0 \leq i_{1}, i_{2} \leq m \\
i_{1}+i_{2}=j}} \int_{0}^{t} v_{i_{1}} \cdot v_{i_{2}}\left(y_{s}^{\mu}, t-s\right) d s\right\}\right\},
\end{aligned}
$$

where $v_{j}(x, t)=\nabla S_{j}(x, t)$.

Remark 2.1. When $T_{0}=1$, the fluid density $\rho$ is simply given by

$$
\rho^{1 / 2}(x, t)=e^{-S_{1}(x, t)} .
$$

In the case $T_{0} \neq 1$, we note that initially $\rho$ is $T_{0}^{2}$ explaining its physical significance. Also, observe that up to the caustic time

$$
\begin{aligned}
\int_{\mathbb{R}^{d}} \rho(x, t) d x & =\int_{\mathbb{R}^{d}} T_{0}^{2}\left(\Phi_{t}^{-1}(x)\right)\left|\operatorname{Det}\left(\nabla_{x} \Phi_{t}^{-1}(x)\right)\right| d x \\
& =\int_{\mathbb{R}^{d}} T_{0}^{2}(y) d y \\
& =\int_{\mathbb{R}^{d}} \rho(y, 0) d y .
\end{aligned}
$$

So mass is conserved.

In this section we have recapitulated some of the key results of $[33,36]$ on stochastic Burgers equations. We have elucidated the detailed structure of the asymptotic series $\theta$ in Equation (1.12) when $\Phi_{s}$ is a diffeomorphism, $0 \leq s \leq t$, so that $x_{0}(x, t)$ is unique. If we now combine the above results with those of Davies and Truman [9, 10], Ellis and Rosen [16, 17, 18], we can see that even when $x_{0}(x, t)$ is not unique, for non degenerate critical points, $\theta_{i}$ has exactly the same structure as above save that we work with the $\mathrm{i}^{\text {th }}$ branch of $\Phi_{t}^{-1}$ defined by $x_{0}^{i}(x, t)$. What breaks down here is the closed form expansion for the remainder term as a functional integral. We hope to address this in a future paper [11] but we will not need those results here. 


\section{Singularities of the Free Burgers Equation and Heat Equation}

We investigate the geometrical relationship between the level surfaces of the heat equation and the shock waves (caustics) of the Burgers equation. We find a robust geometrical relationship between curves on level surfaces and the caustics which (when suitably modified) carries over to the general stochastic set up. In two dimensions the curves on the level surfaces are the level surfaces themselves. In three dimensions we think of these curves as arising from planar cross sections.

Definition 3.1. A curve $x=x(\gamma), \gamma \in N\left(\gamma_{0}, \delta\right)$, is said to have a generalised cusp at $\gamma=\gamma_{0}, \gamma$ being arc length if

$$
\left.\frac{d x(\gamma)}{d \gamma}\right|_{\gamma=\gamma_{0}}=0
$$

\subsection{A Simple Geometrical Relationship}

In this section and Section 5 we were inspired by V. I. Arnol'd's beautiful treatment of classical mechanics in [3].

Let $\mathcal{A}=\mathcal{A}\left(x_{0}, x, t\right)$ be the classical action for the initial momentum $\nabla S_{0}$,

$$
\mathcal{A}\left(x_{0}, x, t\right)=A\left(x_{0}, x, t\right)+S_{0}\left(x_{0}\right),
$$

where in the deterministic case

$$
A\left(x_{0}, x, t\right)=\inf _{\substack{X(s) \\ X(t)=x \\ X(0)=x_{0}}}\left\{\frac{1}{2} \int_{0}^{t} \dot{X}^{2}(s) d s-\int_{0}^{t} c(X(s)) d s\right\},
$$

$c$ being the deterministic potential energy. The Euler-Lagrange equation for the above action reduces to

$$
\ddot{X}(s)=-\nabla c(X(s)), \quad s \in[0, t]
$$

and $X(t)=x, X(0)=x_{0}$. The free case corresponds to setting $c \equiv 0$ and

$$
\begin{aligned}
& A\left(x_{0}, x, t\right)=\frac{\left(x-x_{0}\right)^{2}}{2 t}, \\
& \mathcal{A}\left(x_{0}, x, t\right)=\frac{\left(x-x_{0}\right)^{2}}{2 t}+S_{0}\left(x_{0}\right) .
\end{aligned}
$$


We assume that $\mathcal{A}\left(x_{0}, x, t\right)$ is at least $C^{4}$ in space variables for $t>0$. Defining the corresponding classical flow now reduces to

$$
\nabla_{x_{0}} \mathcal{A}\left(x_{0}, x, t\right)=0 .
$$

i.e.

$$
\left(x_{0}-x\right) / t+\nabla S_{0}\left(x_{0}\right)=0 \quad \text { or } \quad x=\Phi_{t}\left(x_{0}\right)=x_{0}+t \nabla S_{0}\left(x_{0}\right) .
$$

We sometimes write $x_{0}=\Phi_{t}^{-1} x=x_{0}(x, t)$ as the (possibly nonunique) solution of

$$
x_{0}+t \nabla S_{0}\left(x_{0}\right)=x .
$$

Consider the level surface equations obtained by eliminating $x_{0}$ between

$$
\mathcal{A}\left(x_{0}, x, t\right)=0 \quad \text { and } \quad \frac{\partial \mathcal{A}}{\partial x_{0}^{\alpha}}\left(x_{0}, x, t\right)=0, \quad \alpha=1,2, \ldots, d .
$$

We obtain the prelevel surface by eliminating $x$ in the above equations. Considering for any real constant $c$, the equation obtained by eliminating $x_{0}$ between

$$
\mathcal{A}\left(x_{0}, x, t\right)=c \quad \text { and } \quad \frac{\partial \mathcal{A}}{\partial x_{0}^{\alpha}}\left(x_{0}, x, t\right)=0, \quad \alpha=1,2, \ldots, d,
$$

gives the level surface of Hamilton's principal function $S(x, t)=c$ with $S(x, 0)=S_{0}(x)$. We denote this level surface by $H_{t}$ and the prelevel surface by $\Phi_{t}^{-1} H_{t}$.

Imitating the above, the precaustic equation and caustic equation can be obtained in a similar way by considering

$$
\operatorname{Det}\left(\frac{\partial^{2} \mathcal{A}}{\partial x_{0}^{2}}\left(x_{0}, x, t\right)\right)=0, \quad \frac{\partial \mathcal{A}}{\partial x_{0}^{\alpha}}\left(x_{0}, x, t\right)=0, \quad \alpha=1,2, \ldots, d .
$$

We denote these surfaces by $\Phi_{t}^{-1} C_{t}$ and $C_{t}$, respectively.

Let $x \rightarrow \alpha$, a point on the caustic, then, since we expect the multiplicity of the $x_{0}^{i} \in \Phi_{t}^{-1}\{x\}$ to change as we cross the caustic, we anticipate that $x_{0}^{j}(x, t) \rightarrow x_{0}^{k}(x, t)$ as $x \rightarrow \alpha$. Since different parts of the level surface correspond to different $x_{0}^{i}$, if $S_{j}(\alpha, t)=S_{k}(\alpha, t)=c$, then the parts $S_{j}(x, t)=$ $c$ and $S_{k}(x, t)=c$ meeting at $\alpha$ could have a cusp. It is cusps like this which give rise to turbulent behaviour of the Burgers velocity field. We therefore investigate in the free case the conditions under which we get cusps.

In the free case the equation of the zero prelevel surface is the eikonal equation

$$
\frac{t}{2}\left|\nabla S_{0}\left(x_{0}\right)\right|^{2}+S_{0}\left(x_{0}\right)=0
$$


and the derivative map $D \Phi_{t}\left(x_{0}\right): T_{x_{0}} \rightarrow T_{x}$, mapping tangent spaces is the linear map

$$
D \Phi_{t}\left(x_{0}\right)=\left(I+t \nabla^{2} S_{0}\left(x_{0}\right)\right)
$$

where $\nabla^{2} S_{0}$ is the Hessian of $S_{0}$. The following elementary identity is crucial in what follows

$$
\nabla_{x_{0}}\left\{\frac{t}{2}\left|\nabla S_{0}\left(x_{0}\right)\right|^{2}+S_{0}\left(x_{0}\right)\right\}=\left(I+t \nabla^{2} S_{0}\left(x_{0}\right)\right) \nabla S_{0}\left(x_{0}\right) .
$$

Since $D \Phi_{t}\left(x_{0}\right)$ is in this case a real symmetric matrix, we can analyse what happens very easily, especially for two dimensions.

\subsection{Results in Two Dimensions}

Lemma 3.1. Assume that the prelevel surface meets the precaustic at the point $x_{0}$, where $\left|\left(I+t \nabla^{2} S_{0}\left(x_{0}\right)\right) \nabla S_{0}\left(x_{0}\right)\right| \neq 0$ and $\operatorname{dim}\left(\operatorname{Ker}\left(I+t \nabla^{2} S_{0}\left(x_{0}\right)\right)\right)=$ 1. Then the tangent plane to the prelevel surface $T_{x_{0}}$ is spanned by $\operatorname{Ker}(I+$ $\left.t \nabla^{2} S_{0}\left(x_{0}\right)\right)$.

Proof. The last displayed formula shows that where the prelevel surface meets the precaustic the non-zero normal to the prelevel surface is a linear combination of eigenvectors corresponding to the non-zero eigenvalues of $\left(I+t \nabla^{2} S_{0}\left(x_{0}\right)\right)$. The eigenvector of $\left(I+t \nabla^{2} S_{0}\left(x_{0}\right)\right)$ corresponding to eigenvalue zero, $e_{0}$, is orthogonal to these eigenvectors, so because $T_{x_{0}}$ is only one dimensional, we have $T_{x_{0}}=\left\langle e_{0}\right\rangle$.

When $\nabla S_{0}\left(x_{0}\right)=0$ at $x_{0}$ a point of the prelevel surface, the above argument shows that $x_{0}$ is a singular point of the prelevel surface, typically a node with

two different directions of the tangent plane. Because the partial derivatives

$$
\frac{\partial \mathcal{A}}{\partial x_{0}^{\alpha}}\left(x_{0}, x, t\right)=0, \quad \alpha=1,2, \ldots, d
$$

we have for $x_{0}=\Phi_{t}^{-1} x$

$$
\nabla_{x} \mathcal{A}\left(x_{0}, x, t\right)=\frac{\left(x-x_{0}\right)}{t}=\nabla S_{0}\left(x_{0}\right)
$$

independent of dimension. [ In point of fact the last result is true merely because in the free case the momentum is constant ]. Hence, at the image point $x=\Phi_{t}\left(x_{0}\right)$ (independent of the dimensions of the space and independent of whether or not $\left.x_{0} \in \Phi_{t}^{-1} C_{t}\right)\left|\nabla_{x} \mathcal{A}\right|=0$ so $x$ is also a singular point. Typically then, even if we are not on the precaustic, we expect that in the 
free case $x_{0}$ and $\Phi_{t}\left(x_{0}\right)$ are both nodes with two or more different directions of the tangent plane. This eventuality is easy to investigate with the derivative map $D \Phi_{t}\left(x_{0}\right)$. When $x_{0} \in \Phi_{t}^{-1} C_{t}$ (because $D \Phi_{t}\left(x_{0}\right)$ annihilates the zero eigenvector $e_{0}$ on the precaustic) we have only one direction for the tangent plane at $\Phi_{t}\left(x_{0}\right)$ even though there were two distinct directions for the tangent plane to the prelevel surface at $x_{0}$. This happens, for example, where the prelevel surface consists of an ellipse and a line pair crossing at $x_{0}$ where the ellipse is tangential to the parabolic precaustic. It also occurs in the three dimensional Fish and Butterfly example along the straight line of intersection of the caustic and zero level surface. Both of these examples are elucidated in Section 4.

Lemma 3.2. Assume that the prelevel surface meets the precaustic at the point $x_{0}$ where $\left|\nabla S_{0}\left(x_{0}\right)\right|=0, x_{0}$ being a node, and $\operatorname{dim}\left(\operatorname{Ker}\left(I+t \nabla^{2} S_{0}\left(x_{0}\right)\right)\right)=$ 1. Then the image point $\Phi_{t}\left(x_{0}\right)$ where the level surface meets the caustic is a singular point which cannot be a node but can be a cusp. If $x_{0}$ is a node of the prelevel surface, $x_{0} \notin \Phi_{t}^{-1} C_{t}$, then $x=\Phi_{t}\left(x_{0}\right)$ is a node of the level surface.

Proof. In the first case, the two different directions $t_{1}$ and $t_{2}$ at $x_{0}$ of the tangent plane to the prelevel surfaceb where it meets the precaustic are both mapped into $\left(I+t \nabla^{2} S_{0}\left(x_{0}\right)\right) e_{0}^{\perp}, e_{0}^{\perp}$ being the non-zero eigenvector of $\left(I+t \nabla^{2} S_{0}\left(x_{0}\right)\right)$. So both $t_{1}$ and $t_{2}$ get mapped into the same direction as $e_{0}^{\perp}$. Moreover, $|\nabla \mathcal{A}|=0$, so $\Phi_{t}\left(x_{0}\right)$ is a singular point so it has to be a cusp. The second case follows because the two different directions $t_{i}$ get mapped into the two different directions $\left(I+t \nabla^{2} S_{0}\left(x_{0}\right)\right) t_{i}$ for $i=1,2$.

Remark 3.1. Needless to say there is an analogue of this lemma in dimensions. The condition $\left|\nabla S_{0}\left(x_{0}\right)\right|=0$ should be seen as a zero speed condition.

When $\left(I+t \nabla^{2} S_{0}\left(x_{0}\right)\right) \nabla S_{0}\left(x_{0}\right)$ is non-zero, there is a simple result concerning the appearance of cusps on the level surface. We use the intrinsic equation of the prelevel surface $x_{0}=x_{0}(\gamma), \gamma \in N\left(\gamma_{0}, \delta\right)$, where $x_{0}\left(\gamma_{0}\right)$ is the point of intersection of the prelevel surface and precaustic. We write $x(\gamma)=\Phi_{t}\left(x_{0}(\gamma)\right)$ and assume that $x_{0}$ and $x$ are differentiable in $\gamma$.

Proposition 3.1. Assume that $\left|\left(I+t \nabla^{2} S_{0}\left(x_{0}\right)\right) \nabla S_{0}\left(x_{0}\right)\right| \neq 0$, so $x_{0}$ is not a singular point of the prelevel surface. Then $\Phi_{t}\left(x_{0}\right)$ can only be a generalised cusp of the level surface if $\Phi_{t}\left(x_{0}\right) \in C_{t}$, the caustic. Moreover, if $x=\Phi_{t}\left(x_{0}\right) \in$ $\Phi_{t}\left(\Phi_{t}^{-1} C_{t} \cap \Phi_{t}^{-1} H_{t}\right), x$ will indeed be a generalised cusp of the level surface. 
Proof. We have the normal $n\left(x_{0}\right)=\left(I+t \nabla^{2} S_{0}\left(x_{0}\right)\right) \nabla S_{0}\left(x_{0}\right) \neq 0$, $\left.\frac{d x_{0}(\gamma)}{d \gamma}\right|_{\gamma=\gamma_{0}} \neq 0$ and from above

$$
\begin{aligned}
\left.\frac{d x(\gamma)}{d \gamma}\right|_{\gamma=\gamma_{0}} & =\left.\left(D \Phi_{t}\left(x_{0}\right)\right) \frac{d x_{0}(\gamma)}{d \gamma}\right|_{\gamma=\gamma_{0}} \\
& =\left.\left(I+t \nabla^{2} S_{0}\left(x_{0}\right)\right) \frac{d x_{0}(\gamma)}{d \gamma}\right|_{\gamma=\gamma_{0}}
\end{aligned}
$$

For this to be zero it is necessary that $\operatorname{Det}\left(I+t \nabla^{2} S_{0}\left(x_{0}\right)\right)=0$, so $x_{0} \in \Phi_{t}^{-1} C_{t}$ i.e. it is necessary that $\Phi_{t}\left(x_{0}\right) \in C_{t}$. Trivially from Lemma 3.1 it follows that if $x_{0} \in \Phi_{t}^{-1} C_{t}$ then $\left.\frac{d x(\gamma)}{d \gamma}\right|_{\gamma=\gamma_{0}}=0$

\subsection{Results in Three Dimensions}

Consider the above set up in three dimensions so that the wavefront $H_{t}$ has the equation in $x$ obtained by eliminating $x_{0}$ between

$$
\mathcal{A}\left(x_{0}, x, t\right)=c \quad \text { and } \quad \frac{\partial \mathcal{A}}{\partial x_{0}^{\alpha}}\left(x_{0}, x, t\right)=0, \quad \alpha=1,2,3,
$$

and the caustic $C_{t}$ by eliminating $x_{0}$ between

$$
\operatorname{Det}\left(\frac{\partial^{2} \mathcal{A}}{\partial x_{0}^{2}}\left(x_{0}, x, t\right)\right)=0 \quad \text { and } \quad \frac{\partial \mathcal{A}}{\partial x_{0}^{\alpha}}\left(x_{0}, x, t\right)=0, \quad \alpha=1,2,3 .
$$

We define the cusped part of $H_{t}$ by

$$
\begin{aligned}
\operatorname{Cusp}\left(H_{t}\right)= & \left\{x \in H_{t}: x \in \Phi_{t}\left(\Phi_{t}^{-1} C_{t} \cap \Phi_{t}^{-1} H_{t}\right), x=\Phi_{t}\left(x_{0}\right),\right. \\
& \left.x_{0} \in \Phi_{t}^{-1} C_{t} \cap \Phi_{t}^{-1} H_{t}, n\left(x_{0}\right)=\left(I+t \nabla^{2} S_{0}\left(x_{0}\right)\right) \nabla S_{0}\left(x_{0}\right) \neq 0\right\} .
\end{aligned}
$$

Typical of what happens in three dimensions for which $d=3$ is the following:

Theorem 3.1. Let $x \in \operatorname{Cusp}\left(H_{t}\right)$ then $T_{x}$, the tangent plane to the level surface $H_{t}$ at $x$, is at most one dimensional.

Remark 3.2. The tangent plane $T_{x}$ folds over onto itself at points $x \in$ $\operatorname{Cusp}\left(H_{t}\right)$, the direction of the fold being the direction of the one dimensional tangent space at $x$. 
Proof. Let $n$ be the unit normal to the tangent plane $T_{x_{0}}, x_{0} \in \Phi_{t}^{-1} C_{t} \cap$ $\Phi_{t}^{-1} H_{t}$. This is the well defined vector

$$
n=\left|\left(I+t \nabla^{2} S_{0}\left(x_{0}\right)\right) \nabla S_{0}\left(x_{0}\right)\right|^{-1}\left(I+t \nabla^{2} S_{0}\left(x_{0}\right)\right) \nabla S_{0}\left(x_{0}\right),
$$

which is clearly a linear combination of the non-zero eigenvectors of $(I+$ $\left.t \nabla^{2} S_{0}\left(x_{0}\right)\right)$. Let $e_{0}$ be an eigenvector corresponding to eigenvector zero which is orthogonal to $n$. Since $n \neq 0, T_{x_{0}}$ is a well defined two dimensional space spanned by $e_{0}$ and $n \wedge e_{0}=e_{0}^{\perp}$. Because $D \Phi_{t}\left(x_{0}\right)=\left(I+t \nabla^{2} S_{0}\left(x_{0}\right)\right), T_{x}$ is spanned by $\left(I+t \nabla^{2} S_{0}\left(x_{0}\right)\right) e_{0}^{\perp}$.

We now see what it takes for us to observe cusps on planar cross sections of the level surface where it meets the caustic. We shall assume, without loss of generality, that the planar cross sections are on a plane $y=$ constant with normal $e_{y}$. We write $x=\Phi_{t}\left(x_{0}\right)$ and

$$
\nabla S_{0}\left(x_{0}\right)=\alpha_{0} e_{0}+\alpha_{1} e_{1}+\alpha_{2} e_{2}=\nabla_{x} \mathcal{A}
$$

where

$$
\begin{aligned}
& \left(I+t \nabla^{2} S_{0}\left(x_{0}\right)\right) e_{0}=0, \\
& \left(I+t \nabla^{2} S_{0}\left(x_{0}\right)\right) e_{i}=\lambda_{i} e_{i}, \quad i=1,2,
\end{aligned}
$$

$x_{0} \in \Phi_{t}^{-1} C_{t} \cap \Phi_{t}^{-1} H_{t}$.

Then, from above, the unit normal

$$
n=\left(\alpha_{1} \lambda_{1} e_{1}+\alpha_{2} \lambda_{2} e_{2}\right) \theta
$$

and

$$
e_{0}^{\perp}=\left(\alpha_{2} \lambda_{2} e_{1}-\alpha_{1} \lambda_{1} e_{2}\right) \theta,
$$

where $\theta=\left(\alpha_{1}^{2} \lambda_{1}^{2}+\alpha_{2}^{2} \lambda_{2}^{2}\right)^{-\frac{1}{2}}$. At a point $x \in \operatorname{Cusp}\left(H_{t}\right)$, the axis system $\left\{e_{0}, e_{0}^{\perp}, n\right\}$ is well defined and intrinsically related to the geometry of the prelevel surface at $x_{0} \in \Phi_{t}^{-1} C_{t} \cap \Phi_{t}^{-1} H_{t}$. This is our preferred axis system for discussing cusps.

We assume that $S_{0}$ is at least $C^{4}$ and write

$$
x=x_{0}+t \nabla S_{0}\left(x_{0}\right) .
$$

We work to second order

$$
\begin{aligned}
x+\delta x=x_{0} & +\delta x_{0}+t \nabla S_{0}\left(x_{0}+\delta x_{0}\right) \\
=x_{0} & +\delta x_{0}+t \nabla S_{0}\left(x_{0}\right)+t\left(\delta x_{0} \cdot \nabla\right) \nabla S_{0}\left(x_{0}\right)+\frac{t}{2}\left(\delta x_{0} \cdot \nabla\right)^{2} \nabla S_{0}\left(x_{0}\right) \\
& +O\left(\left(\delta x_{0}\right)^{3}\right) .
\end{aligned}
$$


Hence, correct to second order

$$
\delta x=\left(I+t \nabla^{2} S_{0}\left(x_{0}\right)\right) \delta x_{0}+\frac{t}{2}\left(\delta x_{0} \cdot \nabla\right)^{2} \nabla S_{0}\left(x_{0}\right) .
$$

We now set

$$
\delta x_{0}=\varepsilon\left(\lambda e_{0}+\mu e_{0}^{\perp}\right)+\varepsilon^{2}\left(\xi e_{0}+\eta e_{0}^{\perp}+\zeta n\right) .
$$

The first term gives the direction of $d x_{0} / d \gamma$ and the second $d^{2} x_{0} / d \gamma^{2}$ evaluated at $\gamma=\gamma_{0}$, where $x_{0}\left(\gamma_{0}\right)$ is the point of intersection. Then we obtain

$$
\begin{aligned}
\delta x= & \varepsilon \mu \lambda_{1} \lambda_{2}\left(\alpha_{2} e_{1}-\alpha_{1} e_{2}\right) \theta+\varepsilon^{2}\left(\eta \lambda_{1} \lambda_{2}\left(\alpha_{2} e_{1}-\alpha_{1} e_{2}\right) \theta\right. \\
& \left.+\zeta\left(\alpha_{1} \lambda_{1}^{2} e_{1}+\alpha_{2} \lambda_{2}^{2} e_{2}\right) \theta+\frac{t}{2}\left(\lambda \partial_{0}+\mu \partial_{0}^{\perp}\right)^{2} \nabla S_{0}\left(x_{0}\right)\right),
\end{aligned}
$$

$\partial_{0}$ and $\partial_{0}^{\perp}$ being directional derivatives parallel to $e_{0}$ and $e_{0}^{\perp}$.

For a genuine cusp we clearly have to set $\mu=0$, for which

$$
\begin{aligned}
\delta x_{0}=\varepsilon \lambda e_{0}+ & \varepsilon^{2}\left(\xi e_{0}+\eta e_{0}^{\perp}+\zeta n\right), \\
\delta x=\varepsilon^{2}\left(\eta \lambda_{1} \lambda_{2}\left(\alpha_{2} e_{1}-\alpha_{1} e_{2}\right) \theta\right. & \\
& \left.\quad+\zeta\left(\alpha_{1} \lambda_{1}^{2} e_{1}+\alpha_{2} \lambda_{2}^{2} e_{2}\right) \theta+\frac{t}{2} \lambda^{2} \partial_{0}^{2} \nabla S_{0}\left(x_{0}\right)\right) .
\end{aligned}
$$

We now have to satisfy two further conditions:

$$
\begin{aligned}
\delta x \cdot e_{y} & =0 \\
\delta x \cdot \frac{\partial \mathcal{A}}{\partial x} & =\delta x \cdot \nabla S_{0}\left(x_{0}\right) \\
& =\delta x \cdot\left(\alpha_{0} e_{0}+\alpha_{1} e_{1}+\alpha_{2} e_{2}\right)=0 .
\end{aligned}
$$

If we can choose $\lambda, \eta$ and $\zeta$ to satisfy the above two equations for non-zero $\delta x$, the planar cross section of the level surface $H_{t}, y=$ constant, will have a genuine cusp at $x \in \operatorname{Cusp}\left(H_{t}\right)$. The axis of the cusp will be $\widehat{\delta x}$. This explains why genuine cusps are so numerous on planar cross sections.

Theorem 3.2. Any point $x$ on the level surface $H_{t}, x=\Phi_{t}\left(x_{0}\right)$ with $x_{0}=$ $\Phi_{t}^{-1}(x)$ on the prelevel surface, can only be a generalised cusp of a curve on $H_{t}$ if $x_{0}$ is a generalised cusp of the precurve on the prelevel surface or if $x_{0} \in \Phi_{t}^{-1} C_{t}$ the precaustic. In three dimensions, the planar cross section $y=$ constant (with normal $e_{y}$ ) of the level surface $H_{t}$ through a point $x$ where it meets $C_{t}$ will have a genuine cusp at $x$ if $x \in \operatorname{Cusp}\left(H_{t}\right)$ and there is a nonzero solution $\delta x$ of Equations $\left(C_{0}\right),\left(C_{1}\right),\left(C_{2}\right)$ and $\left(C_{3}\right)$. The direction of the axis of the cusp is $\widehat{\delta x}$. 
Proof. The first part of the theorem is obvious.

Equations $\left(C_{1}\right),\left(C_{2}\right)$ and $\left(C_{3}\right)$ uniquely fix the direction of $\widehat{\delta x}$, if non-zero $\lambda$, $\eta$ and $\zeta$ exist satisfying these equations. It easy to see that Equations $\left(C_{0}\right)$, $\left(C_{1}\right),\left(C_{2}\right)$ and $\left(C_{3}\right)$ are sufficient to solve:-

$$
\begin{array}{rlrl}
\mathcal{A}\left(x_{0}, x, t\right) & =c, & \\
\mathcal{A}\left(x_{0}+\delta x_{0}, x+\delta x, t\right) & =c, & \\
\frac{\partial \mathcal{A}}{\partial x_{0}^{\alpha}}\left(x_{0}, x, t\right) & =0, \quad \alpha=1,2,3, \\
\frac{\partial \mathcal{A}}{\partial x_{0}^{\alpha}}\left(\left(x_{0}+\delta x_{0}, x+\delta x, t\right)\right. & =0, \quad \alpha=1,2,3,
\end{array}
$$

correct to second order, if

$$
\operatorname{Det}\left(\left.\frac{\partial^{2} \mathcal{A}}{\partial x_{0}^{2}}\left(x_{0}, x, t\right)\right|_{x=\Phi_{t}\left(x_{0}\right)}\right)=0,
$$

$x_{0} \in \Phi_{t}^{-1} C_{t} \cap \Phi_{t}^{-1} H_{t}, x=\Phi_{t}\left(x_{0}\right), n\left(x_{0}\right)$ non-zero.

This analysis explains the plethora of cusps which we observe in the free case. Moreover, as we shall see, the argument above can be generalised to include random and deterministic external forces i.e. non-zero $c$ and $k$ terms in Burgers equation.

Remark 3.3. We make the point that

$$
\Phi_{t}^{-1} C_{t} \cap \Phi_{t}^{-1} H_{t} \subset \Phi_{t}^{-1}\left(C_{t} \cap H_{t}\right),
$$

but the opposite inclusion does not hold and so images of points in $\Phi_{t}^{-1}\left(C_{t} \cap\right.$ $\left.H_{t}\right) \backslash\left(\Phi_{t}^{-1} C_{t} \cap \Phi_{t}^{-1} H_{t}\right)$ give rise to cross-over points.

\subsection{Consequences for the Burgers Fluid}

Here we recapitulate a result of Truman and Zhao [33]. See [15] for more recent related results.

Theorem 3.3. Let $\tau(a)$ be the first time such that there exist minimisers $y_{1} \neq y_{2}, y_{1}, y_{2} \in \mathbb{R}^{d}$ such that $\Phi_{\tau}\left(y_{1}\right)=\Phi_{\tau}\left(y_{2}\right)=a$ and $\dot{\Phi}_{\tau}\left(y_{1}\right) \neq \dot{\Phi}_{\tau}\left(y_{2}\right)$, i.e. $\tau$ is a caustic time at point $a$. Then $v^{0}(x, t)$ is discontinuous at $(\tau, a)$.

We now give a simple application of the above results to the Burgers fluid in two dimensions. For smooth $S_{0}$, in two dimensions, we can divide one side of the caustic into hot and cool parts. We consider points of intersection $\alpha$ with 
the level surface $S(x, t)=S(\alpha, t)$ cusped at $\alpha$ : if the part of the level surface cusped at $\alpha$ corresponds to the minimising $x_{0}(\alpha, t)$ then the Burgers velocity field $v^{0}(x, t)=\nabla S(x, t) \rightarrow \nabla S(\alpha, t)=0$ as $x \rightarrow \alpha$ from the cusped side of the caustic. This is merely because the condition for $S(x, t)=S(\alpha, t)$ to have a cusp at $x=\alpha$ is $\nabla S(\alpha, t)=0$. Moreover, this entails the minimising surface changing as we cross the caustic. Indeed the cusp occurs because the minimising surface on the cusped side of the caustic cannot be continued across the caustic. So $u^{0}(x, t)$ is necessarily exponentially discontinuous as we cross such parts of the caustic. This is because here two $x_{0}(x, t)$ 's coalesce as the minimiser at the cusp and then disappear. We call such points $\alpha$ cool because the Burgers fluid has zero velocity on one side of the caustic at $\alpha$. The whole of one side of the generic semi-cubical parabolic Cusp is cool. Not all of one side of the two dimensional Swallowtail is cool. This is discussed in the next section, where we give numerical confirmation of this result. The exponential discontinuity in $u^{0}$ can be seen by inspecting the following diagram giving the number of negative $S_{0}^{i}$ 's in the different regions. Similar results apply in three dimensions.

Figure 10. Number of Negative $S_{0}$

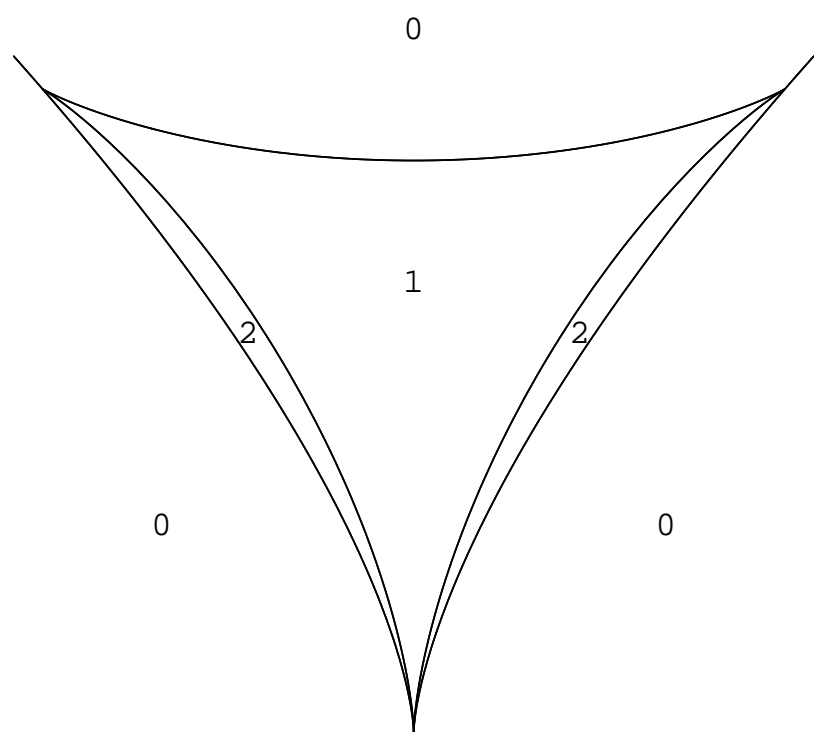




\section{Deterministic and Archetypal Examples}

Consider $S_{0}$ given in the following table for two and three dimensions respectively,

\begin{tabular}{|l|l|c|}
\hline Shockwave & Zero Level Surface & $S_{0}$ \\
\hline Cusp & Tricorn & $x_{0}^{2} y_{0} / 2$ \\
\hline Swallowtail & Plough & $x_{0}^{5}+\left|x_{0}\right|^{3 / 2} y_{0}$ \\
\hline Butterfly & Fish & $x_{0}^{3} y_{0} / 3+x_{0}^{2} z_{0} / 2$ \\
\hline Swallowtail & Seagull & $\left|x_{0}\right|^{5}+\left|x_{0}\right|^{1+c} y_{0}+\left|x_{0}\right|^{c} z_{0}$ \\
\hline
\end{tabular}

where $\frac{1}{2}<c<1$. The nature of the caustic (shockwave) is given in the first column and the corresponding zero level surfaces in the second. The zero level surface corresponding to the semicubical parabolic Cusp is the Tricorn, which has three cusps on the semi-cubical parabola, one of which coincides with the original cusp of the shockwave, and a line-pair. The zero level surface corresponding to the Butterfly is the Fish which meets the Butterfly in four curves (three of which are cusped and one of which is a straight line on which they touch). See $[31,32]$ for further and more surprising examples.

\subsection{A two dimensional example}

For $S_{0}\left(x_{0}, y_{0}\right)=x_{0}^{2} y_{0} / 2$ the zero prelevel surface has equation

$$
x_{0}^{2}\left(\frac{\left(y_{0}+\frac{1}{2 t}\right)^{2}}{\left(\frac{1}{2 t}\right)^{2}}+\frac{x_{0}^{2}}{\left(\frac{1}{t}\right)^{2}}-1\right)=0,
$$

and the precaustic has equation

$$
1+t y_{0}=t^{2} x_{0}^{2}
$$

Note also that $\nabla S_{0}\left(x_{0}, y_{0}\right)=0$ at the point $\left(0,-\frac{1}{t}\right)$ so the zero speed condition is satisfied here and this point is a node of the zero prelevel surface. We now map $(x, y)=\Phi_{t}\left(x_{0}, y_{0}\right)$ to obtain the hypocycloid tricorn

$$
x=\frac{\cos \theta(1+\sin \theta)}{2 t}, \quad y=\frac{\sin \theta(1-\sin \theta)}{2 t},
$$

for $0 \leq \theta<2 \pi$, as the zero level surface at time $t$ for the heat equation and semi-cubical parabolic cusp

$$
8(y t+1)^{3}=27 t^{2} x^{2}
$$

as shockwave at time $t$ for the corresponding Burgers velocity field. 
The level surface $S_{t}(x, y)=0$.

Figure 11. Precaustic and Prelevel Surface

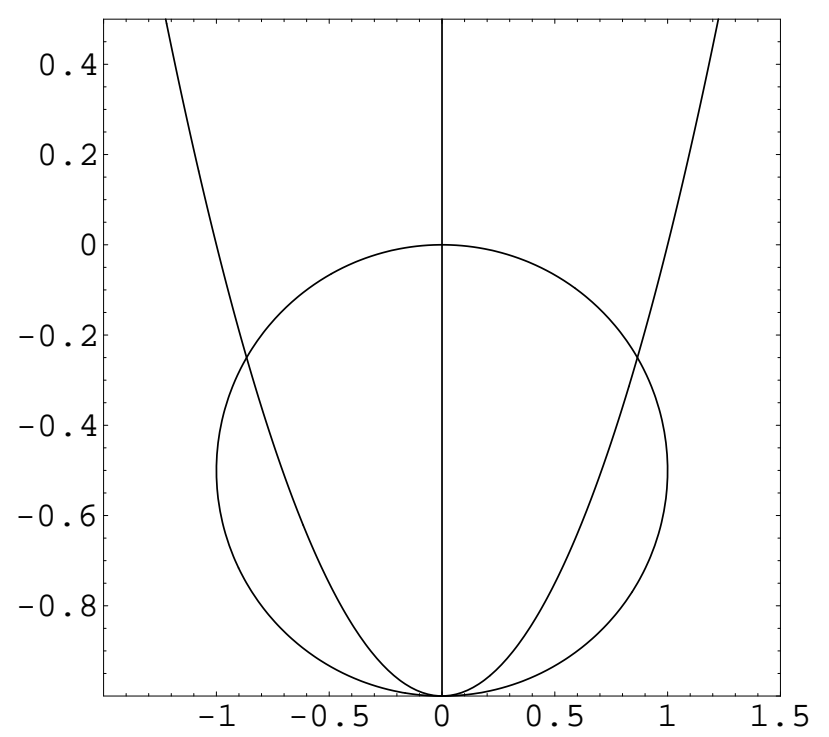

Figure 12. Cusp and Tricorn

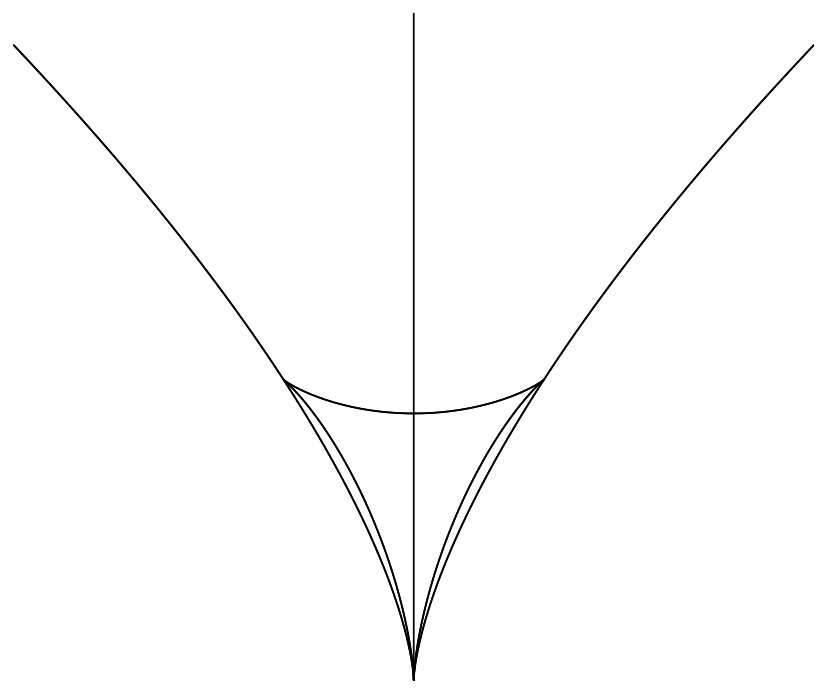


The level surface $S_{t}(x, y)=2$

Figure 13. Precaustic (Parabola) and Prelevel Surface (Kneecap)

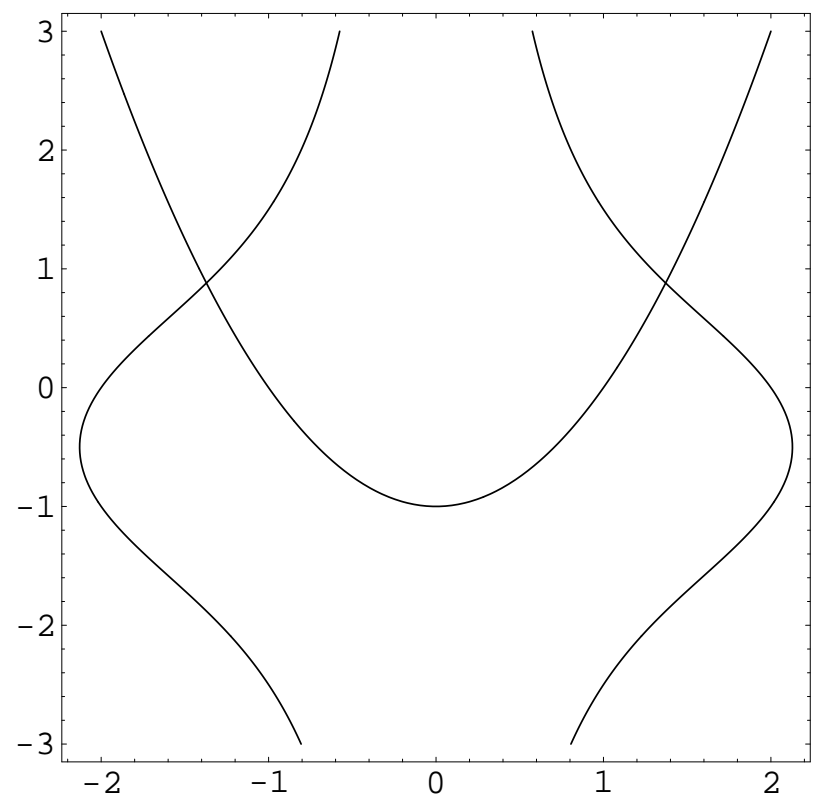

Figure 14. Caustic (Cusp) and Level Surface (Neckline)

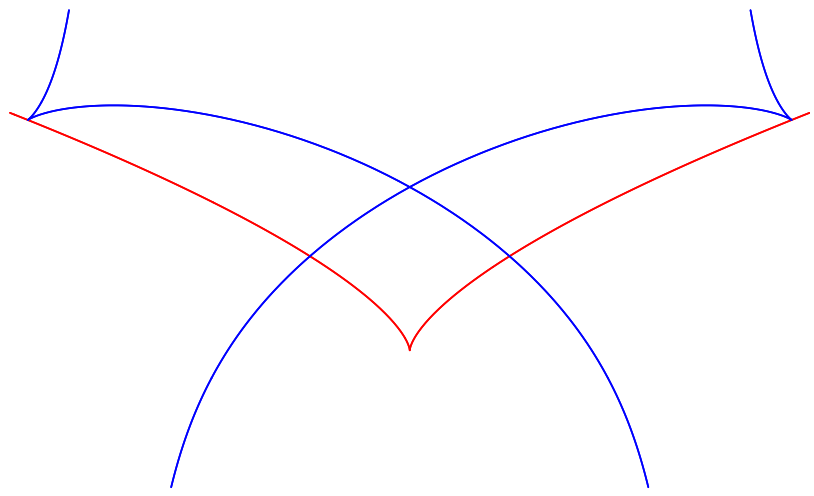


The level surface $S_{t}(x, y)=-2^{-7}$

Figure 15. Precaustic (Parabola) and Prelevel Surface (Pebble Pair)

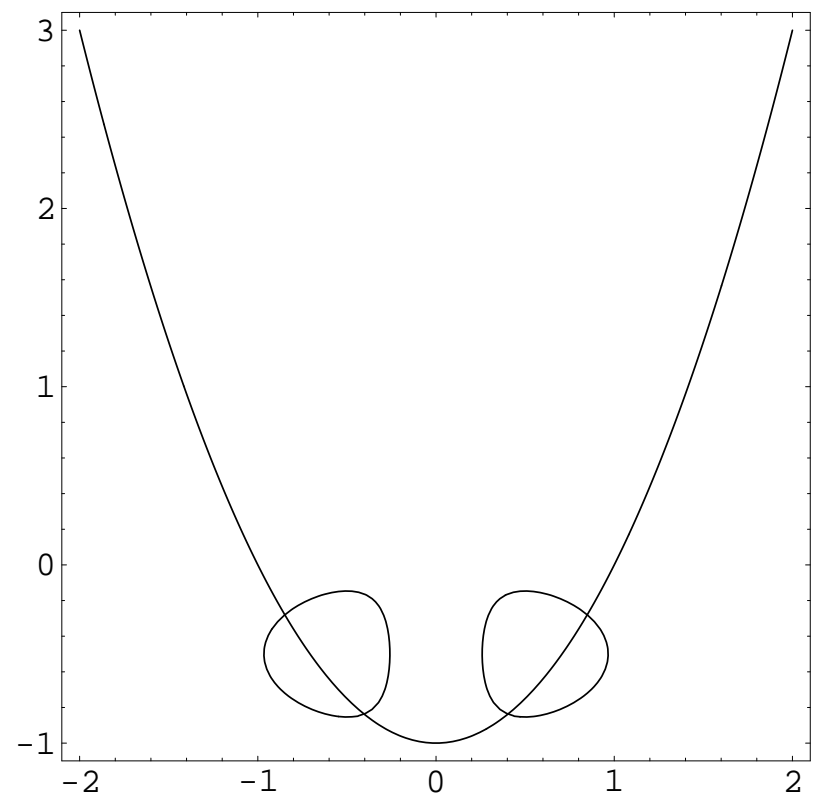

Figure 16. Caustic (Cusp) and Level Surface (Lapel Pair)

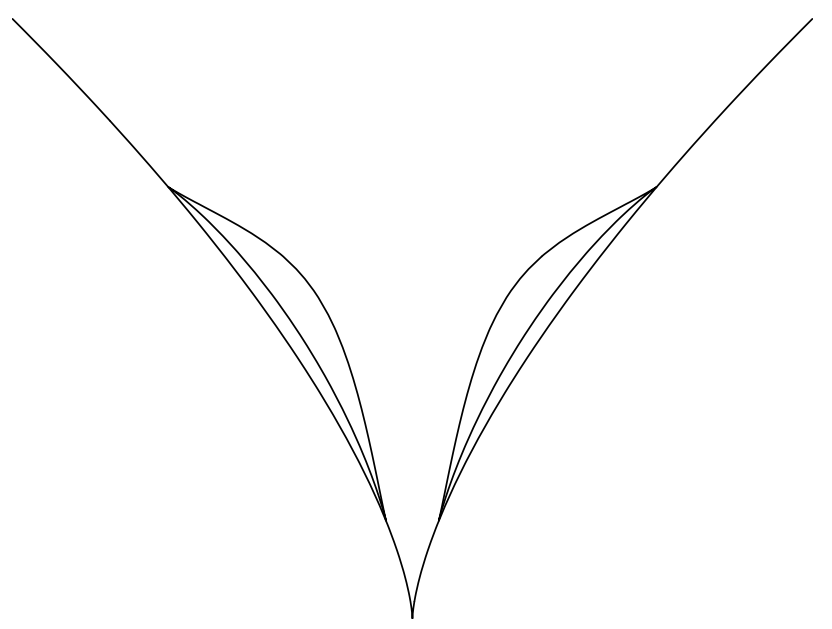


We have verified numerically that the caustic surfaces are indeed discontinuities for the Burgers velocity field as shown below. Further, it is not difficult to see that the whole of one side of this Cusp surface is cool. One has to note, and it is not too onerous to check, that for each point of the cusp the minimising $S_{0}^{i}$ corresponds to a cusp and not a crossover.

Figure 17. $\left|v^{\mu}\right|^{2}$ for small $\mu$

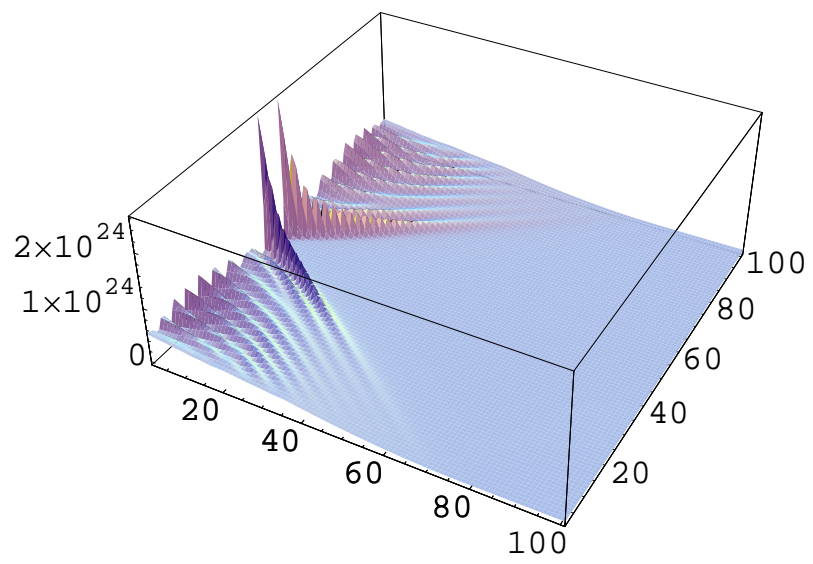

Figure 18. Contour Plot of $\left|v^{\mu}\right|^{2}$ with Cusp and Tricorn

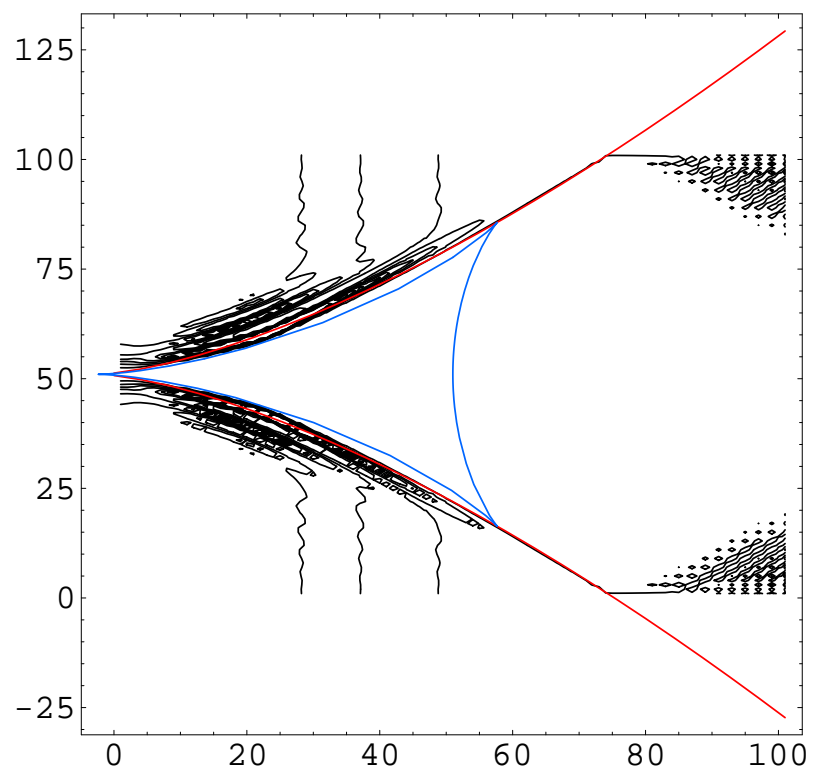

Finally we add that in two dimensions the Tricorn will be a common feature of the level surfaces of Hamilton's principal function when the corresponding 
prelevel surface touches the precaustic. Here the point is that the unfolding of the Tricorn under the inverse classical flow map $\Phi_{t}$ is the 'halo' shown in Figure 11. The point of contact is denoted by $x_{0}^{0}$ and the direction of the unit tangent vector by $e_{0}$. To see this from above we know that $\Phi_{t}\left(x_{0}^{0} \pm \varepsilon e_{0}\right)=$ $\Phi_{t}\left(x_{0}^{0}\right)+\mathrm{o}(\varepsilon)$. If the slightly stronger result holds, namely $\Phi_{t}\left(x_{0}^{0} \pm \varepsilon e_{0}\right)=$ $\Phi_{t}\left(x_{0}^{0}\right)+\varepsilon^{2} w_{0}+\mathrm{o}\left(\varepsilon^{2}\right)$, then necessarily we have a tricorn with axis $w_{0}$ since both halves of the common tangent get mapped onto the half axis parallel to $w_{0}$. The other two points of intersection being mapped onto cusps. 


\subsection{A three dimensional example}

For $S_{0}\left(x_{0}, y_{0}, z_{0}\right)=x_{0}^{3} y_{0} / 3+x_{0}^{2} z_{0} / 2$ we can quickly see the close relationship with the previous example by means of the cross-sections $y=$ constant shown below.

Figure 19. Pre - Butterfly and Fish

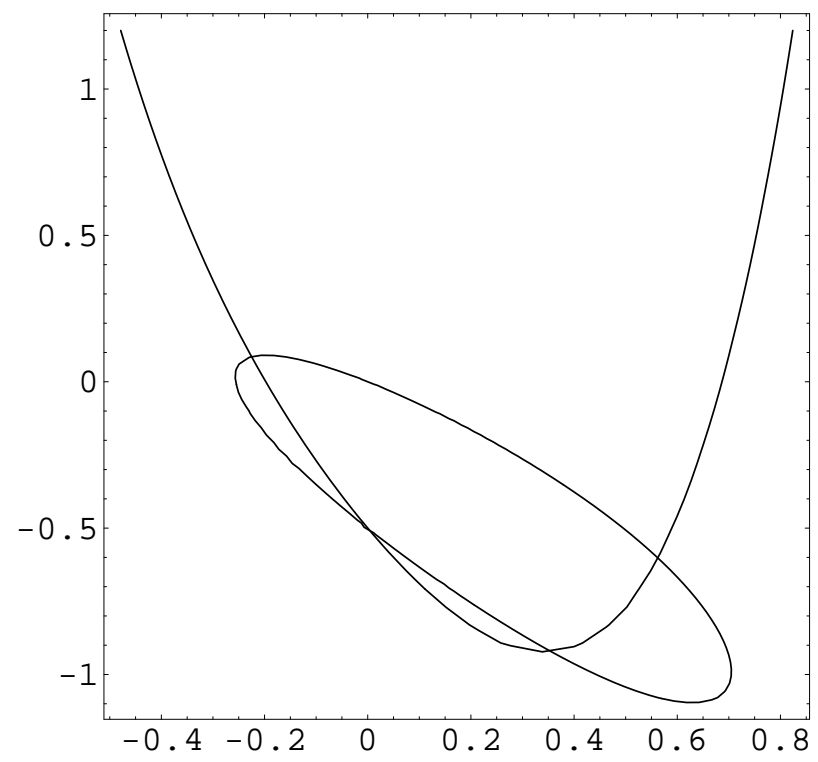

Figure 20. Butterfly and Fish

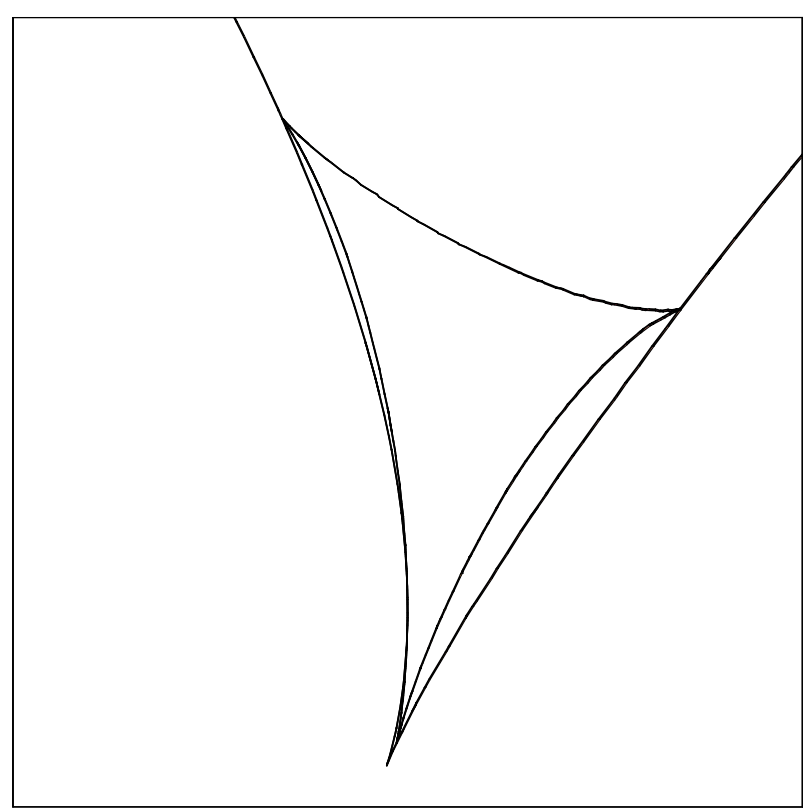


To see where the Butterfly and Fish meet, consider the classical mechanics:

$$
\begin{aligned}
& x=x_{0}+t\left(x_{0}^{2} y_{0}+x_{0} z_{0}\right), \\
& y=y_{0}+\frac{t}{3} x_{0}^{3}, \\
& z=z_{0}+\frac{t}{2} x_{0}^{2} .
\end{aligned}
$$

Solving the precaustic equation leads to

$$
z_{0}=-\frac{1-t^{2} x_{0}^{2}-t^{2} x_{0}^{4}+2 t x_{0} y_{0}}{t}
$$

and with this we simplify the zero prelevel surface equation, one solution of which is $x_{0}=0$ leaving a quadratic equation for $y_{0}$ with roots,

$$
y_{0}=\frac{-2+6 t^{2} x_{0}^{2}+6 t^{2} x_{0}^{4}-\sqrt{4+3 t^{2} x_{0}^{2}+8 t^{2} x_{0}^{4}}}{6 t x_{0}},
$$

and

$$
y_{0}=\frac{-2+6 t^{2} x_{0}^{2}+6 t^{2} x_{0}^{4}+\sqrt{4+3 t^{2} x_{0}^{2}+8 t^{2} x_{0}^{4}}}{6 t x_{0}} .
$$

When $x_{0}=0$, we have $z_{0}=-\frac{1}{t}$ and $\nabla S_{0}\left(x_{0}, y_{0}, z_{0}\right)=0$ on this line. Hence on this line the zero speed condition is satisfied and the tangent plane to the zero prelevel surface here has two possible directions. Therefore the zero level surface and caustic meet on a straight line at

$$
x=0, z=-1 / t, y \in \mathbb{R} .
$$

It is easy to see that the two surfaces actually touch on this line because this line is on the precaustic. For one value of $y_{0}$, we obtain the parametric equations of the two curves of intersection $\left(x_{0}>0\right.$ and $\left.x_{0}<0\right)$

$$
\begin{aligned}
& x=\frac{1}{6} x_{0}\left(2+\sqrt{4+t^{2} x_{0}^{2}\left(3+8 x_{0}^{2}\right)}\right), \\
& y=-\frac{2-2 t^{2} x_{0}^{2}\left(3+4 x_{0}^{2}\right)+\sqrt{4+t^{2} x_{0}^{2}\left(3+8 t^{2}\right)}}{6 t x_{0}}, \\
& z=\frac{-3 t^{2} x_{0}^{2}\left(1+2 x_{0}^{2}\right)+2\left(-1+\sqrt{4+t^{2} x_{0}^{2}\left(3+8 t^{2}\right)}\right)}{6 t} .
\end{aligned}
$$


For the other value of $y_{0}$ we obtain the parametric equation of one additional curve

$$
\begin{aligned}
& x=-\frac{1}{6} x_{0}\left(-2+\sqrt{4+t^{2} x_{0}^{2}\left(3+8 x_{0}^{2}\right)}\right), \\
& y=\frac{-2+2 t^{2} x_{0}^{2}\left(3+4 x_{0}^{2}\right)+\sqrt{4+t^{2} x_{0}^{2}\left(3+8 t^{2}\right)}}{6 t x_{0}}, \\
& z=-\frac{3 t^{2} x_{0}^{2}\left(1+2 x_{0}^{2}\right)+2\left(1+\sqrt{4+t^{2} x_{0}^{2}\left(3+8 t^{2}\right)}\right)}{6 t} .
\end{aligned}
$$

Figure 21. The Butterfly Singularity for Burgers Equation

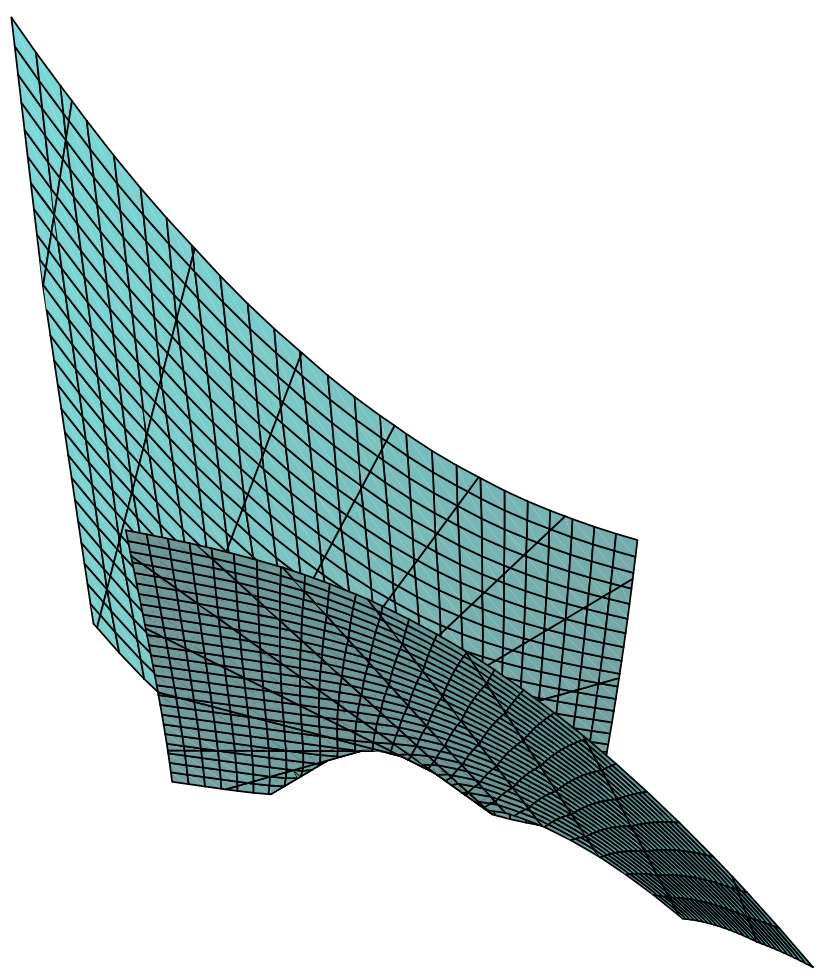


Figure 22. The Fish Level Surface for Heat Equation (a section)

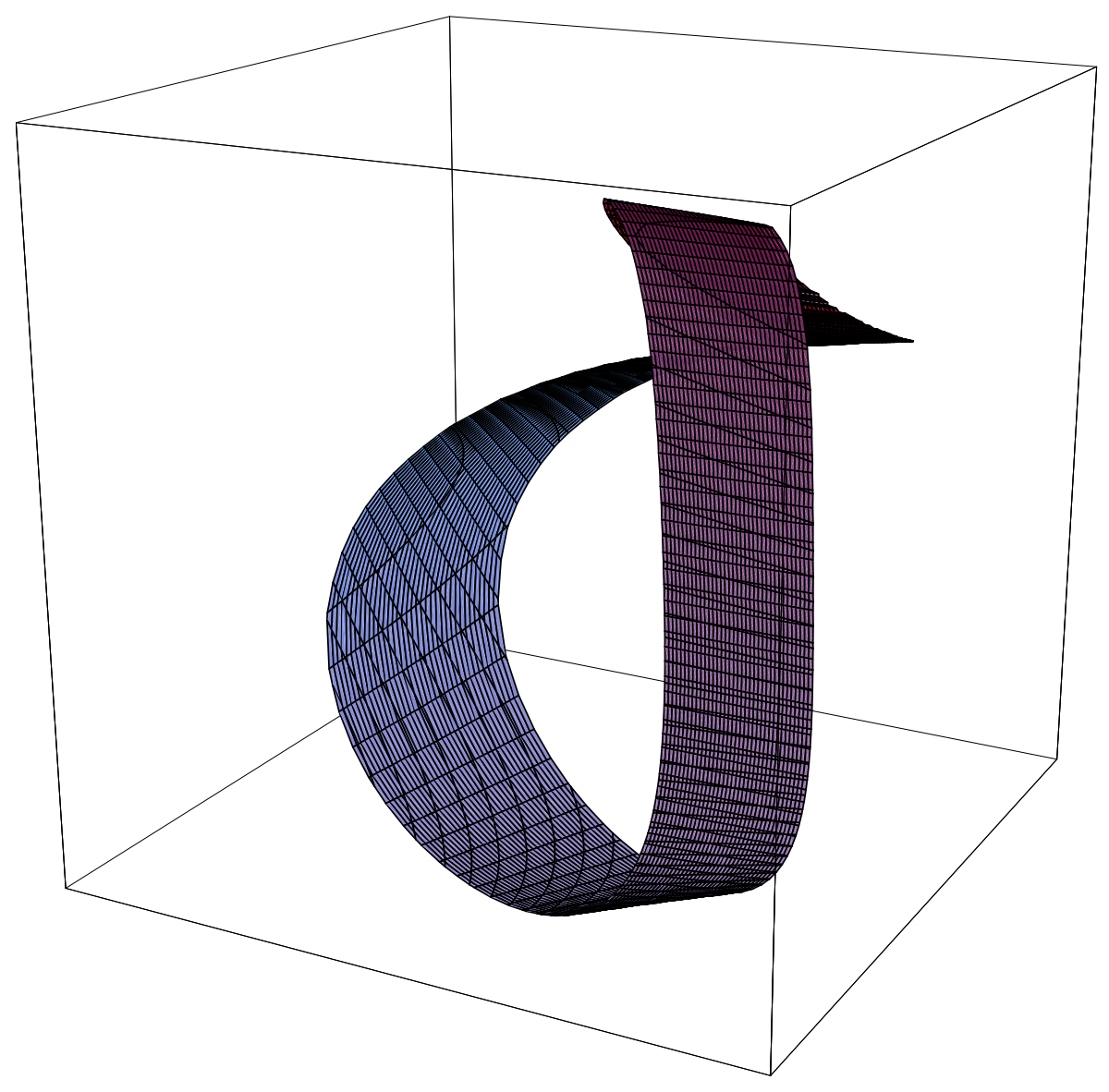




\subsection{A cautionary two dimensional example}

We briefly consider the non-generic two dimensional swallowtail, where $S_{0}\left(x_{0}, y_{0}\right)=x_{0}^{5}+\left|x_{0}\right|^{\frac{3}{2}} y_{0}$. Here the zero level surface crosses the caustic because $\Phi_{t}^{-1}\left(C_{t} \cap H_{t}\right) \not \subset \Phi_{t}^{-1} C_{t} \cap \Phi_{t}^{-1} H_{t}$. This example also shows that we can have $\frac{\left.d x_{0}\left(\gamma_{0}\right)\right)}{d \gamma}=0$ occurring on the zero prelevel surface although we should point out that $S_{0}$ is non-polynomial in this case. We have many other cases of polynomial $S_{0}$ giving rise to two-dimensional swallowtail singularities.

Figure 23. Precaustic

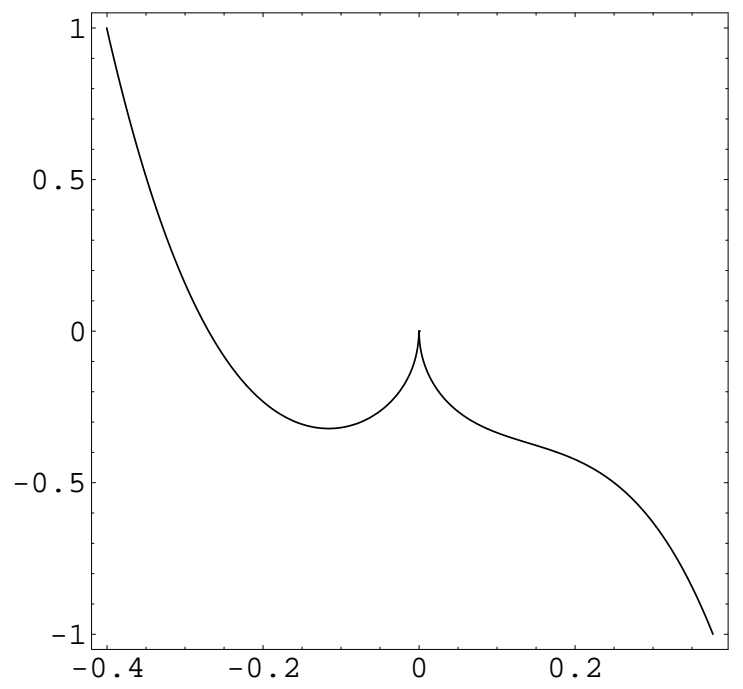

Figure 24. Prelevel Surface

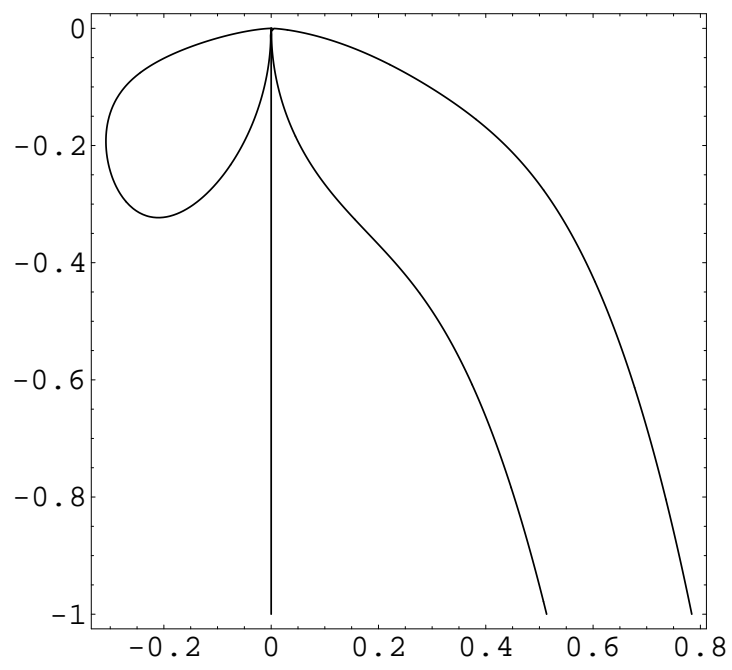


Note the alignment of the generalised cusps on the zero prelevel surface and precaustic.

Figure 25. Swallowtail Caustic

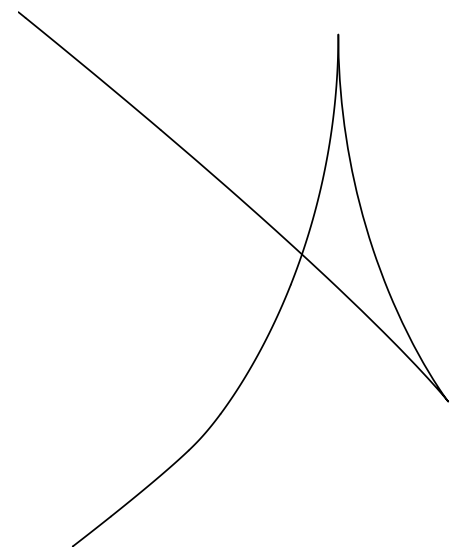

Figure 26. Plough Level Surface

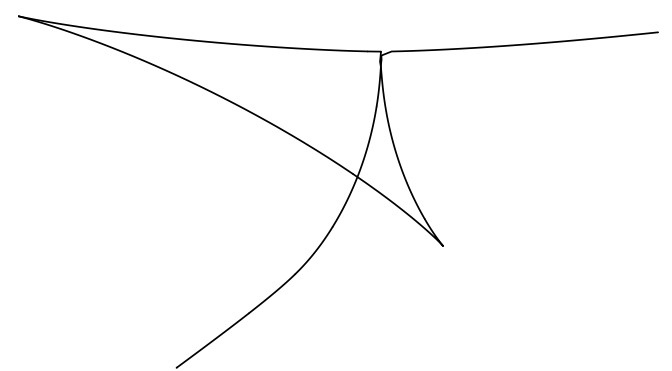

We also illustrate the case where the zero level surface crosses the caustic, at the points $P$ and $Q$, when $\Phi_{t}^{-1}$ gives distinct points on the zero prelevel surface and precaustic.

Figure 27. Swallowtail and Plough

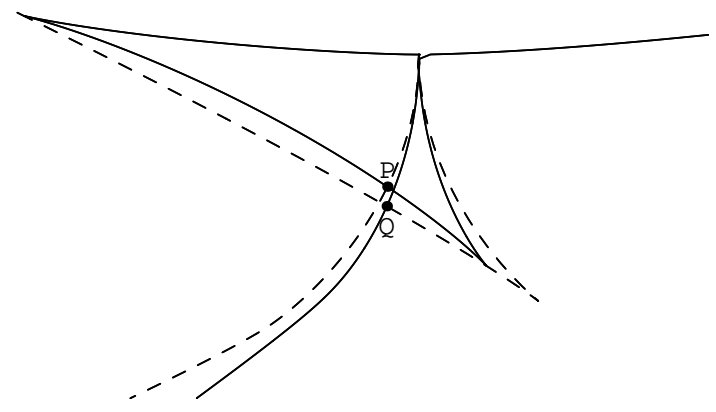




\subsection{Swallowtail}

We briefly illustrate the caustic for $S_{0}\left(x_{0}, y_{0}, z_{0}\right)=\left|x_{0}\right|^{5}+\left|x_{0}\right|^{5 / 3} y_{0}+\left|x_{0}\right|^{2 / 3} z_{0}$. This was the first such $S_{0}$ found to generate the Swallowtail but we also have examples of strictly polynomial $S_{0}$ which generate the same archetypal caustic in three dimensions in analogy to the examples of the previous section.

Figure 28. The Swallowtail Singularity for Burgers Equation

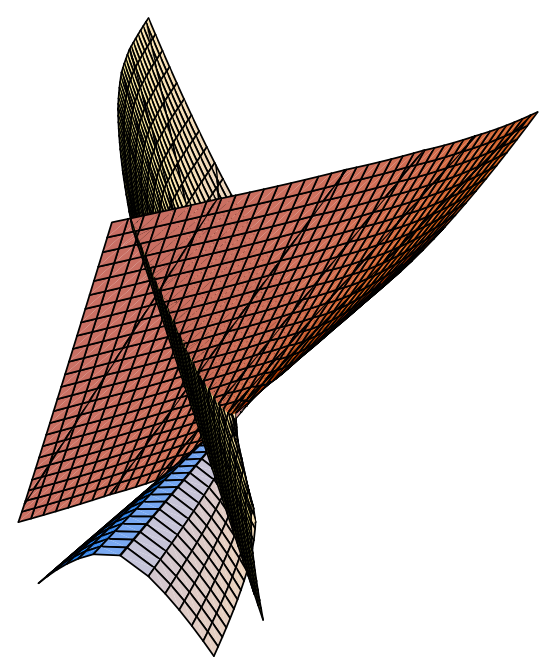

Figure 29. The Seagull Level Surface for Heat Equation

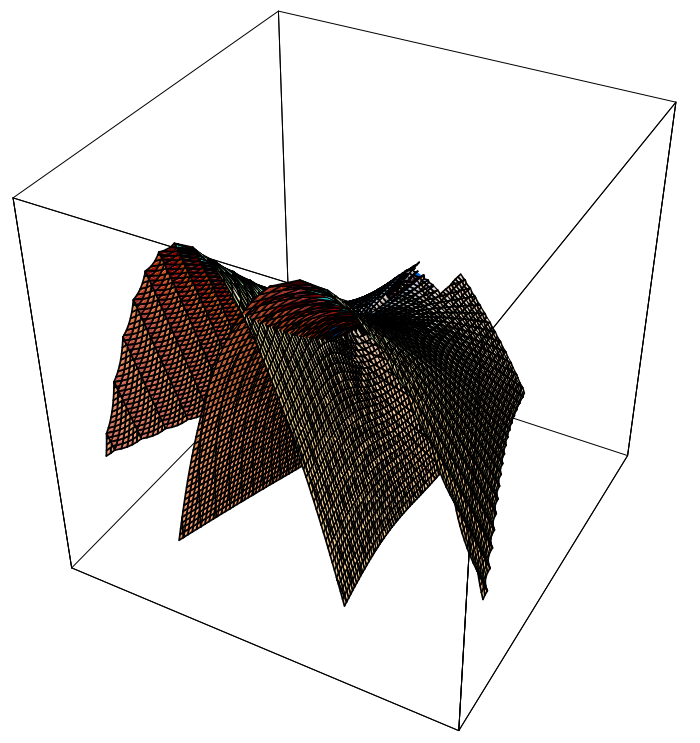




\section{The Stochastic and General Cases}

\subsection{The Stochastic Action}

Let $A\left(x_{0}, p_{0}, t\right)$ be defined by

$$
A\left(x_{0}, p_{0}, t\right)=\frac{1}{2} \int_{0}^{t} \dot{X}^{2}(s) d s-\int_{0}^{t}\left[c(X(s)) d s+\epsilon k(X(s), s) d W_{s}\right],
$$

almost surely, where $X(s)=X_{s}=X\left(s, x_{0}, p_{0}\right)$ satisfies

$$
d \dot{X}(s)=-\nabla c(X(s)) d s-\epsilon \nabla k(X(s), s) d W_{s}, \quad s \in[0, t],
$$

with $X(0)=x_{0}$ and $\dot{X}(0)=p_{0}, x_{0}, p_{0} \in \mathbb{R}^{d}$. We shall assume that $X_{s}$ is unique and as usual is $\mathcal{F}_{s}$ measurable. We also allow for $p_{0}$ to be an as yet unspecified function of $x_{0}$.

Recall the integration by parts property $\left(\mathrm{ibp}^{2}\right)$ for the continuous process $\dot{X}(s)=\dot{X}\left(s, x_{0}, p_{0}\right)$,

$$
\int_{0}^{t} u(s) d \dot{X}(s)=u(t) \dot{X}(t)-u(0) \dot{X}(0)-\int_{0}^{t} \dot{u}(s) \dot{X}(s) d s
$$

for deterministic $u \in C^{1}(0, t)$. This integration by parts property is also valid for random $u$, if the stochastic integral is of Itô's form and, $d u_{s} d \dot{X}_{s}$, the corresponding Itô correction is zero. An important case in point is when

$$
u_{s}=\frac{\partial X_{s}}{\partial x_{0}^{\alpha}},
$$

for $s \in[0, t]$ and $\alpha=1,2, \ldots, d$. Then, if we assume $\nabla c, \nabla k$ are Lipschitz, with Hessians $\nabla^{2} c, \nabla^{2} k$ and all second derivatives with respect to space variables of $c$ and $k$ bounded, according to Kunita [27], $\partial X_{s} / \partial x_{0}^{\alpha}$ satisfies

$$
\frac{d}{d s}\left(\frac{\partial X_{s}}{\partial x_{0}^{\alpha}}\right)=\frac{\partial \dot{X}_{0}}{\partial x_{0}^{\alpha}}-\int_{0}^{s}\left[\nabla^{2} c(X(r)) \frac{\partial X(r)}{\partial x_{0}^{\alpha}} d r+\epsilon \nabla^{2} k(X(r), r) \frac{\partial X(r)}{\partial x_{0}^{\alpha}} d W_{r}\right]
$$

So the Itô correction is zero. Hence for $u_{s}=\partial X_{s} / \partial x_{0}^{\alpha}$ we can use the ibp ${ }^{2}$. Moreover,

$$
\dot{X}_{s}=\dot{X}_{0}-\int_{0}^{s}\left[\nabla c(X(r)) d r+\epsilon \nabla k(X(r), r) d W_{r}\right]
$$

Therefore, using Kunita [27] again, we obtain

$$
\frac{d}{d s}\left(\frac{\partial X_{s}}{\partial x_{0}^{\alpha}}\right)=\frac{\partial \dot{X}_{s}}{\partial x_{0}^{\alpha}} \quad \alpha=1,2, \ldots, d .
$$

This gives : 
Lemma 5.1. Assume $S_{0}, c \in C^{2}$ and $k \in C^{2,0}, \nabla c, \nabla k$ are Lipschitz, with Hessians $\nabla^{2} c, \nabla^{2} k$ and all second derivatives with respect to space variables of $c$ and $k$ bounded. If $\dot{X}_{s}$ satisfies Equation (5.1) and $p_{0}$, possibly $x_{0}$ dependent, then almost surely

$$
\frac{\partial A}{\partial x_{0}^{\alpha}}\left(x_{0}, p_{0}, t\right)=\dot{X}(t) \cdot \frac{\partial X(t)}{\partial x_{0}^{\alpha}}-\dot{X}_{\alpha}(0)
$$

Proof. Arguing as above, for $\alpha=1,2, \ldots, d$, we obtain

$$
\begin{aligned}
\frac{\partial A}{\partial x_{0}^{\alpha}}\left(x_{0}, p_{0}, t\right) & =\int_{0}^{t}\left(\dot{X}(s) \cdot \frac{\partial \dot{X}(s)}{\partial x_{0}^{\alpha}}-\nabla c(X(s)) \cdot \frac{\partial X(s)}{\partial x_{0}^{\alpha}}\right) d s \\
& -\epsilon \int_{0}^{t} \nabla k(X(s), s) \cdot \frac{\partial X(s)}{\partial x_{0}^{\alpha}} d W_{s} .
\end{aligned}
$$

So using the key identity and the $\mathrm{ibp}^{2}$ formula gives

$$
\begin{aligned}
\frac{\partial A}{\partial x_{0}^{\alpha}}\left(x_{0}, p_{0}, t\right)=- & \int_{0}^{t} \frac{\partial X(s)}{\partial x_{0}^{\alpha}} \cdot\left[d \dot{X}(s)+\nabla c(X(s)) d s+\epsilon \nabla k(X(s), s) d W_{s}\right] \\
& +\left[\dot{X}(s) \cdot \frac{\partial X(s)}{\partial x_{0}^{\alpha}}\right]_{0}^{t},
\end{aligned}
$$

proving the desired result.

Remark 5.1. Observe that, if we fix $X(t)$, we obtain almost surely

$$
\frac{\partial A}{\partial x_{0}^{\alpha}}\left(x_{0}, p_{0}, t\right)=-\dot{X}_{\alpha}(0)
$$

for $\alpha=1,2, \ldots, d$.

Let

$$
X\left(s, x_{0}, x\right)=\left.X\left(s, x_{0}, p_{0}\right)\right|_{p_{0}=p\left(x_{0}, x, t\right)},
$$

where $p_{0}=p\left(x_{0}, x, t\right)$ is the (random) minimiser (assumed unique) of $A\left(x_{0}, p_{0}, t\right)$ with $X\left(t, x_{0}, p_{0}\right)=x$. Set

$$
A\left(x_{0}, x, t\right)=\left.A\left(x_{0}, p_{0}, t\right)\right|_{p_{0}=p\left(x_{0}, x, t\right)} .
$$

Theorem 5.1. Defining $A\left(x_{0}, x, t\right)$ as above

$$
\left.\frac{\partial}{\partial x_{0}^{\alpha}}\right|_{\text {fixed }(x, t)} A\left(x_{0}, x, t\right)=-\dot{X}_{\alpha}(0), \quad \alpha=1,2, \ldots, d,
$$

and so

$$
\left.\frac{\partial}{\partial x_{0}^{\alpha}}\right|_{\text {fixed }(x, t)}\left[A\left(x_{0}, x, t\right)+S_{0}\left(x_{0}\right)\right]=0, \quad \alpha=1,2, \ldots, d,
$$

defines the classical stochastic mechanical flow map $\Phi_{t}$ with $x=\Phi_{t}\left(x_{0}\right)$. 
Proof. A trivial consequence of the last lemma.

We now define the stochastic action corresponding to the initial momentum $\nabla S_{0}\left(x_{0}\right)$ by

$$
\mathcal{A}\left(x_{0}, x, t\right)=A\left(x_{0}, x, t\right)+S_{0}\left(x_{0}\right) .
$$

\subsection{The Level Surface Geometry}

We assume that $\mathcal{A}\left(x_{0}, x, t\right)$ is $C^{4}$ in space variables and that $t$ is such that

$$
\operatorname{Det}\left(\frac{\partial^{2} \mathcal{A}}{\partial x_{0} \partial x}\left(x_{0}, x, t\right)\right) \neq 0, \quad x_{0}, x \in \mathbb{R}^{d} .
$$

The last assumption can be considerably weakened but with this last assumption the proofs are simpler. In the free case, considered previously, this assumption is valid for all $t>0$.

We now imitate the free case. We define the prelevel surface of Hamilton's principal function by eliminating $x$ between the equations

$$
\mathcal{A}\left(x_{0}, x, t\right)=c \quad \text { and } \quad \frac{\partial \mathcal{A}}{\partial x_{0}^{\alpha}}\left(x_{0}, x, t\right)=0, \quad \alpha=1,2, \ldots, d,
$$

and the level surface by eliminating $x_{0}$. So the prelevel surface is $\Phi_{t}^{-1} H_{t}$. Similarly we define the caustic $C_{t}$ and the precaustic $\Phi_{t}^{-1} C_{t}$ by eliminating $x_{0}$ or $x$ between

$$
\operatorname{Det}\left(\frac{\partial^{2} \mathcal{A}}{\partial x_{0}^{2}}\left(x_{0}, x, t\right)\right)=0 \quad \text { and } \quad \frac{\partial \mathcal{A}}{\partial x_{0}^{\alpha}}\left(x_{0}, x, t\right)=0, \quad \alpha=1,2, \ldots, d \text {. }
$$

Lemma 5.2. The classical flow map $x=\Phi_{t}\left(x_{0}\right)$ is a differentiable map from $\Phi_{t}^{-1} H_{t}$ to $H_{t}$ with Frechet derivative

$$
D \Phi_{t}\left(x_{0}\right)=\left(-\frac{\partial^{2} \mathcal{A}}{\partial x \partial x_{0}}\left(x_{0}, x, t\right)\right)^{-1}\left(\frac{\partial^{2} \mathcal{A}}{\partial x_{0}^{2}}\left(x_{0}, x, t\right)\right)
$$

if $\mathcal{A}$ is $C^{3}$ in space derivatives.

Proof. We assume $x=\Phi_{t}\left(x_{0}\right), x_{0} \in \Phi_{t}^{-1} H_{t}, x \in H_{t}$, so

$$
\mathcal{A}\left(x_{0}, x, t\right)=c \quad \text { and } \quad \frac{\partial \mathcal{A}}{\partial x_{0}^{\alpha}}\left(x_{0}, x, t\right)=0, \quad \alpha=1,2, \ldots, d .
$$

We move to a neighbouring point $\left(x_{0}+\delta x_{0}, x+\delta x, t\right)$ on $\Phi_{t}^{-1} H_{t}$ and $H_{t}$ respectively. Then we obtain

$$
\mathcal{A}\left(x_{0}+\delta x_{0}, x+\delta x, t\right)=c \quad \text { and } \quad \frac{\partial \mathcal{A}}{\partial x_{0}^{\alpha}}\left(x_{0}+\delta x_{0}, x+\delta x, t\right)=0, \quad \alpha=1,2, \ldots, d .
$$


i.e. correct to first order

$$
\left(\frac{\partial^{2} \mathcal{A}}{\partial x_{0}^{2}}\right) \delta x_{0}+\left(\frac{\partial^{2} \mathcal{A}}{\partial x \partial x_{0}}\right) \delta x=0
$$

and

$$
\delta x \cdot \frac{\partial \mathcal{A}}{\partial x}\left(x_{0}, x, t\right)=0
$$

proving the desired result.

We can now prove the following proposition in $d$ dimensions.

Proposition 5.1. We consider the random prelevel surface obtained by eliminating $x$ between the equations

$$
\mathcal{A}\left(x_{0}, x, t\right)=c \quad \text { and } \quad \frac{\partial \mathcal{A}}{\partial x_{0}^{\alpha}}\left(x_{0}, x, t\right)=0, \quad \alpha=1,2, \ldots, d .
$$

Then the normal to the prelevel surface at the point $x_{0}$ is to within a scalar multiplier given by

$$
n\left(x_{0}\right)=-\left(\frac{\partial^{2} \mathcal{A}}{\partial x_{0}^{2}}\right)\left(\frac{\partial^{2} \mathcal{A}}{\partial x_{0} \partial x}\right)^{-1} \dot{X}\left(t, x_{0}, \nabla S_{0}\left(x_{0}\right)\right) .
$$

Proof. Evidently $n\left(x_{0}\right)=\nabla_{x_{0}} \mathcal{A}\left(x_{0}, x, t\right)$ where $x=\Phi_{t}\left(x_{0}\right)$, the map $\Phi_{t}$ being defined by

$$
\frac{\partial \mathcal{A}}{\partial x_{0}^{\alpha}}\left(x_{0}, x, t\right)=0, \quad \alpha=1,2, \ldots, d .
$$

Hence only the partial derivatives with respect to $x$ contribute to $n\left(x_{0}\right)$ giving

$$
n=\left.\left(D \Phi_{t}\left(x_{0}\right)\right)^{T} \frac{\partial A}{\partial x}\left(x_{0}, x, t\right)\right|_{x=\Phi_{t}\left(x_{0}\right)},
$$

$T$ denoting transpose. However, following the method of Lemma 5.1, we obtain

$$
\left.\frac{\partial A}{\partial x^{\alpha}}\left(x_{0}, x, t\right)\right|_{x=\Phi_{t}\left(x_{0}\right)}=\dot{X}_{\alpha}\left(t, x_{0}, \nabla S_{0}\left(x_{0}\right)\right),
$$

almost surely, for $\alpha=1,2, \ldots, d$, proving the proposition.

Corollary 5.1. In three dimensions at any point $x_{0} \in \Phi_{t}^{-1} C_{t} \cap \Phi_{t}^{-1} H_{t}$ where $n\left(x_{0}\right) \neq 0$ and

$$
\left.\operatorname{Ker}\left(\frac{\partial^{2} \mathcal{A}}{\partial x_{0}^{2}}\left(x_{0}, x, t\right)\right)\right|_{x=\Phi_{t}\left(x_{0}\right)}=\left\langle e_{0}\right\rangle,
$$

$e_{0}$ being the zero eigenvector, $T_{x_{0}}$ the tangent plane to the prelevel surface is spanned by $e_{0}$ and $\left(n\left(x_{0}\right) \wedge e_{0}\right)$. 
Proof. We obtain by symmetry of $\left(\frac{\partial^{2} \mathcal{A}}{\partial x_{0}^{2}}\right)$

$$
e_{0} \cdot n=-\left(\frac{\partial^{2} \mathcal{A}}{\partial x_{0}^{2}}\right) e_{0} \cdot\left(\frac{\partial^{2} \mathcal{A}}{\partial x_{0} \partial x}\right)^{-1} \dot{X}\left(t, x_{0}, \nabla S_{0}\left(x_{0}\right)\right)=0
$$

So $e_{0}$ is in the tangent plane $T_{x_{0}}$. Setting $e_{0}^{\perp}=n \wedge e_{0} \in T_{x_{0}}$ by definition.

Remark 5.2. When the speed $\left|\dot{X}\left(t, x_{0}, \nabla S_{0}\left(x_{0}\right)\right)\right|=0, n\left(x_{0}\right)=0$ and so $x_{0}$ is a singular point of the prelevel surface, either a node with two distinct directions for the tangent plane or a cusped singularity. Because $\frac{\partial \mathcal{A}}{\partial x}=\dot{X}$ necessarily $x=\Phi_{t}\left(x_{0}\right)$ is a singular point of the level surface even if $x_{0} \notin$ $\Phi_{t}^{-1} C_{t}$. In the case $x_{0} \notin \Phi_{t}^{-1} C_{t}$ and $\dot{X}=0$ we know that this singularity can only be a node because the tangent space is fully two dimensional at this point.

Corollary 5.2. In two dimensions let the prelevel surface meet the precaustic at a point $x_{0}$ where $n\left(x_{0}\right) \neq 0$ and $\operatorname{Ker}\left(\frac{\partial^{2} \mathcal{A}}{\partial x_{0}^{2}}\left(x_{0}, \Phi_{t}\left(x_{0}\right), t\right)\right)=\left\langle e_{0}\right\rangle$, $e_{0}$ being the zero eigenvector. Then the tangent plane to the prelevel surface at $x_{0}$, $T_{x_{0}}$ is spanned by $e_{0}$.

Proof. This is a trivial consequence of the proposition above.

Proposition 5.2. Assume that in two dimensions at $x_{0} \in \Phi_{t}^{-1} H_{t}$ the above normal $n\left(x_{0}\right) \neq 0$, so that the prelevel surface does not have a generalised cusp at $x_{0}$. Then the level surface can only have a generalised cusp at $\Phi_{t}\left(x_{0}\right)$ if $\Phi_{t}\left(x_{0}\right) \in C_{t}$, the caustic surface. Moreover, if $x=\Phi_{t}\left(x_{0}\right) \in$ $\Phi_{t}\left\{\Phi_{t}^{-1} C_{t} \cap \Phi_{t}^{-1} H_{t}\right\}$, the level surface will have a generalised cusp at $x$.

Proof. Because $n\left(x_{0}\right)$ is non-zero, the direction of the tangent to the prelevel surface curve is well defined at $x_{0}$. Hence, if the intrinsic parameterisation of the curve is $x_{0}=x_{0}(\gamma), \gamma \in N\left(\gamma_{0}, \delta\right)$ in a neighbourhood of $x_{0}=x_{0}\left(\gamma_{0}\right)$, the tangent to the level surface

$$
\begin{aligned}
\left.\frac{d x(\gamma)}{d \gamma}\right|_{\gamma=\gamma_{0}} & =\left(\left.D \Phi_{t}\left(x_{0}\right) \frac{d x_{0}(\gamma)}{d \gamma}\right|_{\gamma=\gamma_{0}}\right) \\
& =\left(-\frac{\partial^{2} \mathcal{A}}{\partial x \partial x_{0}}\right)^{-1}\left(\frac{\partial^{2} \mathcal{A}}{\partial x_{0}^{2}}\right) \frac{d x_{0}\left(\gamma_{0}\right)}{d \gamma} \\
& =0
\end{aligned}
$$

because $T_{x_{0}}$ is spanned by $e_{0}, x_{0} \in \Phi_{t}^{-1} H_{t} \cap \Phi_{t}^{-1} C_{t}$. What is more $\frac{d x\left(\gamma_{0}\right)}{d \gamma}=0$ implies that necessarily Det $\left(\frac{\partial^{2} \mathcal{A}}{\partial x_{0}^{2}}\right)=0$ if $x_{0}\left(\gamma_{0}\right)$ is not a generalised cusp. 
Needless to say, if $\frac{d x_{0}\left(\gamma_{0}\right)}{d \gamma}=0$ it follows from the above that $\frac{d x\left(\gamma_{0}\right)}{d \gamma}=0$. So generalised cusps map into generalised cusps regardless of whether or not $x_{0} \in \Phi_{t}^{-1} C_{t}$, c.f. Figures 23 and 24 for the two dimensional swallowtail. This is true independent of the dimension of the space. We now return to the three dimensional set up.

Definition 5.1. We define the cusped part of the level surface $H_{t}$ in three dimensions by

$$
\operatorname{Cusp}\left(H_{t}\right)=\left\{x \in H_{t}: x \in \Phi_{t}\left(\Phi_{t}^{-1} C_{t} \cap \Phi_{t}^{-1} H_{t}\right), x=\Phi_{t}\left(x_{0}\right), n\left(x_{0}\right) \neq 0\right\} .
$$

Proposition 5.3. Let $x \in \operatorname{Cusp}\left(H_{t}\right)$ then in three dimensions, $T_{x}$, the tangent space to the level surface at $x$ is one dimensional at most.

Proof. We know that $x=\Phi_{t}\left(x_{0}\right), x_{0} \in \Phi_{t}^{-1} C_{t} \cap \Phi_{t}^{-1} H_{t}$ with $n\left(x_{0}\right) \neq 0$. It follows that there is a well defined two dimensional tangent plane to the prelevel surface at $x_{0}, T_{x_{0}}$ spanned by $e_{0}$ and $\left(n \wedge e_{0}\right)$. However, the derivative map is given by

$$
D \Phi_{t}\left(x_{0}\right)=\left(-\frac{\partial^{2} \mathcal{A}}{\partial x \partial x_{0}}\right)^{-1}\left(\frac{\partial^{2} \mathcal{A}}{\partial x_{0}^{2}}\right) .
$$

So $D \Phi_{t}\left(x_{0}\right) e_{0}=0$ and $T_{x}=\left\langle D \Phi_{t}\left(x_{0}\right)\left(n \wedge e_{0}\right)\right\rangle$ is at most one dimensional.

Of course a similar result holds in higher dimensions.

\subsection{A Plethora of Cusps}

We conclude by explaining why we expect to see generalised cusps on planar cross sections of level surfaces in three dimensions. We first of all observe that, if $\delta x$ is of second order of small quantities, solving

$$
\frac{\partial \mathcal{A}}{\partial x_{0}^{\alpha}}\left(x_{0}, x, t\right)=0, \quad \alpha=1,2,3,
$$

and

$$
\frac{\partial \mathcal{A}}{\partial x_{0}^{\alpha}}\left(x_{0}+\delta x_{0}, x+\delta x, t\right)=0, \quad \alpha=1,2,3,
$$

to second order reduces in our case to

$$
\delta x=\left(-\frac{\partial^{2} \mathcal{A}}{\partial x \partial x_{0}}\right)^{-1}\left\{\left(\frac{\partial^{2} \mathcal{A}}{\partial x_{0}^{2}}\right) \delta x_{0}+\frac{1}{2}\left(\delta x_{0} \cdot \nabla_{x_{0}}\right)^{2} \frac{\partial \mathcal{A}}{\partial x_{0}}\right\} .
$$

Write

$$
\left(\frac{\partial^{2} \mathcal{A}}{\partial x_{0} \partial x}\right)^{-1} \dot{X}\left(t, x_{0}, \nabla S_{0}\left(x_{0}\right)\right)=-\left(\alpha_{0} e_{0}+\alpha_{1} e_{1}+\alpha_{2} e_{2}\right)
$$


where $e_{0}$ is the zero eigenvector of $\left(\frac{\partial^{2} \mathcal{A}}{\partial x_{0}^{2}}\right)$ and

$$
\frac{\partial^{2} \mathcal{A}}{\partial x_{0}^{2}} e_{i}=\lambda_{i} e_{i}, \quad i=1,2
$$

are the remaining eigenvector equations, $\left\{e_{0}, e_{1}, e_{2}\right\}$ an orthonormal system. Then, from above, unit normal

$$
n=\left(\alpha_{1} \lambda_{1} e_{1}+\alpha_{2} \lambda_{2} e_{2}\right)\left(\alpha_{1}^{2} \lambda_{1}^{2}+\alpha_{2}^{2} \lambda_{2}^{2}\right)^{-\frac{1}{2}}
$$

and

$$
e_{0}^{\perp}=n \wedge e_{0}=\left(\alpha_{2} \lambda_{2} e_{1}-\alpha_{1} \lambda_{1} e_{2}\right)\left(\alpha_{1}^{2} \lambda_{1}^{2}+\alpha_{2}^{2} \lambda_{2}^{2}\right)^{-\frac{1}{2}} .
$$

We now set

$$
\delta x_{0}=\varepsilon\left(\lambda e_{0}+\mu e_{0}^{\perp}\right)+\varepsilon^{2}\left(\xi e_{0}+\eta e_{0}^{\perp}+\zeta n\right) .
$$

The first term gives the direction of $\frac{d x_{0}\left(\gamma_{0}\right)}{d \gamma}$, the second term $\frac{d^{2} x_{0}\left(\gamma_{0}\right)}{d \gamma^{2}}$. Then

$$
\begin{array}{r}
\delta x=\left(-\frac{\partial^{2} \mathcal{A}}{\partial x \partial x_{0}}\right)^{-1}\left\{\left[\varepsilon \mu \lambda_{1} \lambda_{2}\left(\alpha_{2} e_{1}-\alpha_{1} e_{2}\right)+\varepsilon^{2}\left(\eta \lambda_{1} \lambda_{2}\left(\alpha_{2} e_{1}-\alpha_{1} e_{2}\right)\right.\right.\right. \\
\left.\left.\left.+\zeta\left(\alpha_{1} \lambda_{1}^{2} e_{1}+\alpha_{2} \lambda_{2}^{2} e_{2}\right)\right)\right] \theta+\frac{1}{2}\left(\delta x_{0} \cdot \nabla_{x_{0}}\right)^{2} \frac{\partial \mathcal{A}}{\partial x_{0}}\right\}
\end{array}
$$

where $\theta=\left(\alpha_{1}^{2} \lambda_{1}^{2}+\alpha_{2}^{2} \lambda_{2}^{2}\right)^{-\frac{1}{2}}$. Note that there is an $\varepsilon^{2}$ term hidden within the last term of the above.

Clearly for a genuine cusp we have to set $\mu=0$ giving

$$
\begin{aligned}
& \delta x_{0}=\varepsilon \lambda e_{0}+ \varepsilon^{2}\left(\xi e_{0}+\eta e_{0}^{\perp}+\zeta n\right) \\
& \delta x=\left(-\frac{\partial^{2} \mathcal{A}}{\partial x \partial x_{0}}\right)^{-1}\left\{\varepsilon ^ { 2 } \theta \left(\eta \lambda_{1} \lambda_{2}\left(\alpha_{2} e_{1}-\alpha_{1} e_{2}\right)\right.\right. \\
&\left.\left.\quad+\zeta\left(\alpha_{1} \lambda_{1}^{2} e_{1}+\alpha_{2} \lambda_{2}^{2} e_{2}\right)\right)+\frac{\varepsilon^{2}}{2} \lambda^{2} \partial_{0}^{2} \frac{\partial \mathcal{A}}{\partial x_{0}}\right\}
\end{aligned}
$$

We now have to satisfy two further conditions:

$$
\begin{aligned}
\delta x \cdot e_{y}=0, & \\
\delta x \cdot \frac{\partial \mathcal{A}}{\partial x}=\left(\alpha_{0} e_{0}\right. & \left.+\alpha_{1} e_{1}+\alpha_{2} e_{2}\right) \cdot\left(\theta \left(\eta \lambda_{1} \lambda_{2}\left(\alpha_{2} e_{1}-\alpha_{1} e_{2}\right)\right.\right. \\
& \left.\left.+\zeta\left(\alpha_{1} \lambda_{1}^{2} e_{1}+\alpha_{2} \lambda_{2}^{2} e_{2}\right)\right)+\frac{\lambda^{2}}{2} \partial_{0}^{2} \frac{\partial \mathcal{A}}{\partial x_{0}}\right)=0 .
\end{aligned}
$$


Surprisingly the last condition does not depend upon $\eta$. It simplifies to give

$$
\left.\zeta\left(\alpha_{1}^{2} \lambda_{1}^{2}+\alpha_{2}^{2} \lambda_{2}^{2}\right)\right)^{\frac{1}{2}}+\frac{\lambda^{2}}{2}\left(\alpha_{0} e_{0}+\alpha_{1} e_{1}+\alpha_{2} e_{2}\right) \cdot \partial_{0}^{2} \frac{\partial \mathcal{A}}{\partial x_{0}}=0
$$

The equation $\left(C_{3}^{\prime}\right)$ determines the ratio $\zeta: \lambda^{2}$ and equation $\left(C_{2}\right)$ determines the ratio $\eta: \lambda^{2}$.

Theorem 5.2. Any point $x$ on the level surface $H_{t}, x=\Phi_{t}\left(x_{0}\right)$ with $x_{0}=$ $\Phi_{t}^{-1}(x)$ on the prelevel surface, can only be a generalised cusp of a curve on $H_{t}$ if $x_{0}$ is a generalised cusp of the precurve on the prelevel surface or if $x_{0} \in \Phi_{t}^{-1} C_{t}$ the precaustic. In three dimensions the planar cross section $y=$ constant (with normal $e_{y}$ ) of the level surface $H_{t}$ through a point $x$ where it meets $C_{t}$ the caustic surface, will have a genuine cusp at $x$ if $x \in \operatorname{Cusp}\left(H_{t}\right)$ and there is a non-zero solution $\delta x$ of Equations $\left(C_{1}\right),\left(C_{2}\right)$ and $\left(C_{3}\right)$. The direction of the axis of the cusp will be $\widehat{\delta x}$.

Proof. Similar to that of Theorem 3.2 using the generalisations expounded above. 


\subsection{A Random Example}

Example 5.1. Here we illustrate the random caustic and level surface for the case $S_{0}\left(x_{0}, y_{0}\right)=x_{0}^{2} y_{0} / 2, c(x)=0$ and $k(x, t)=x$ for $\epsilon=\frac{1}{10}$. The first two figures correspond to $S_{t}(x, y, \omega)=0$.

Figure 30. Stochastic Precaustic and Prelevel Surface

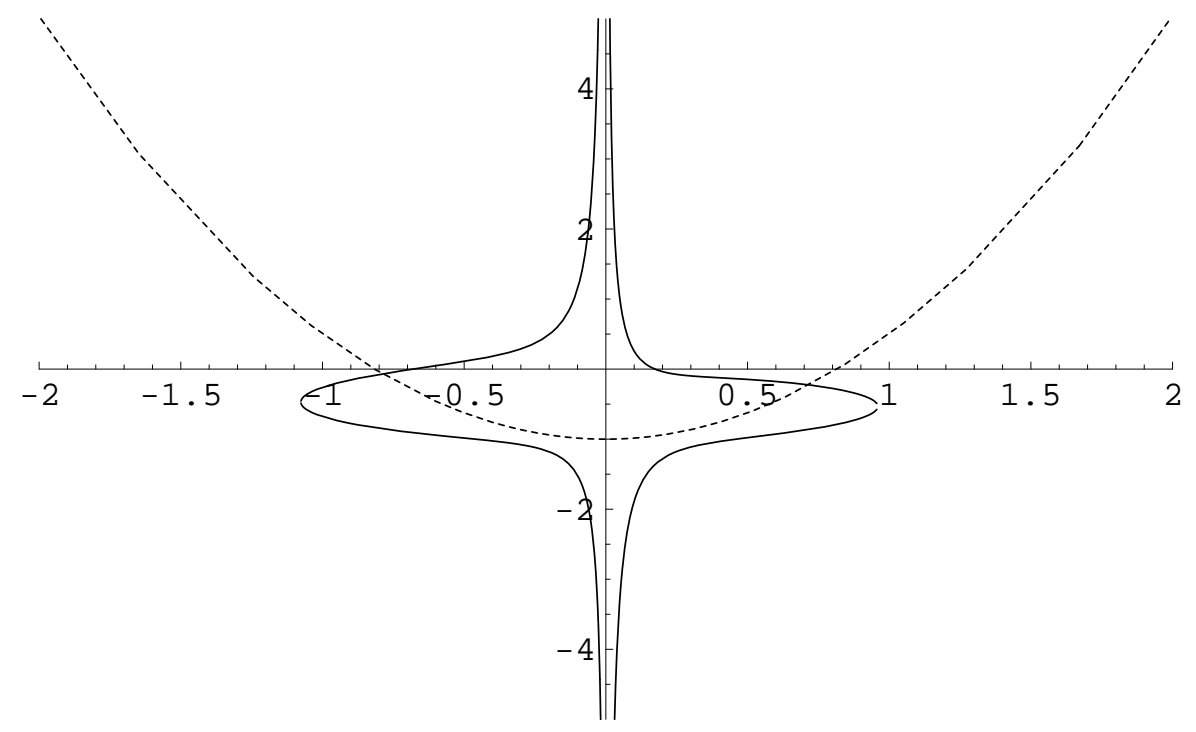

Figure 31. Stochastic Caustic and Level Surface

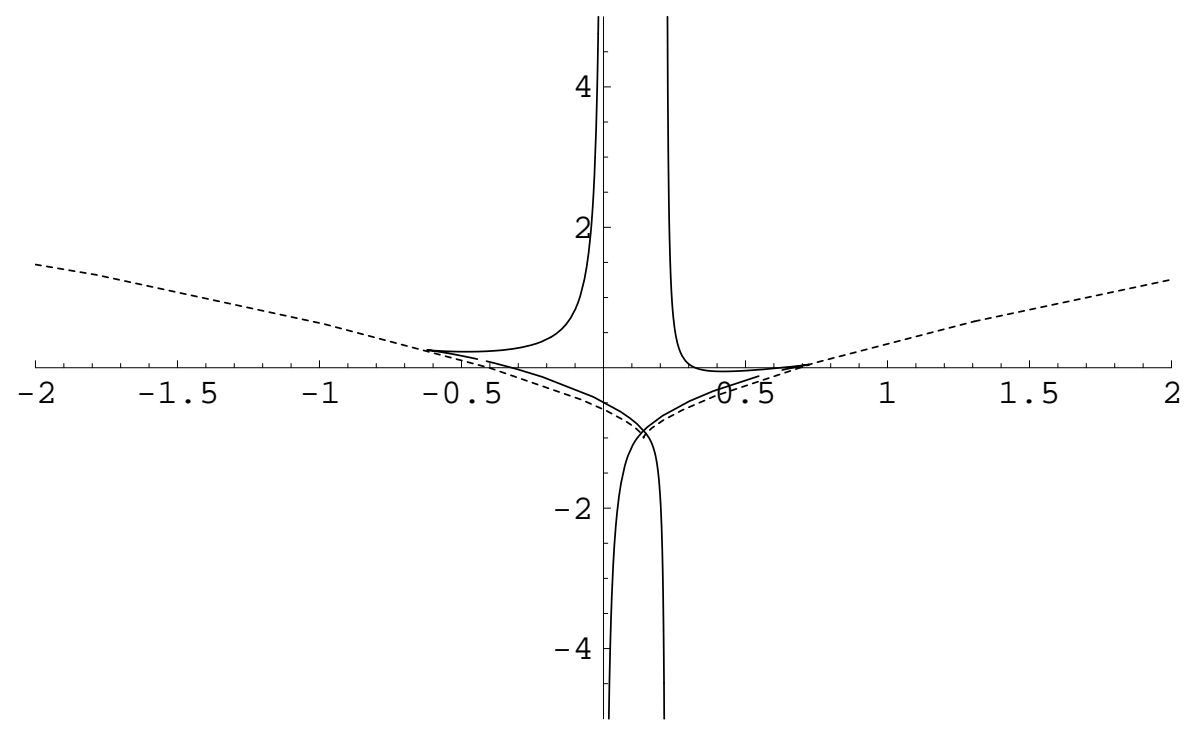


We now display the figures corresponding to $S_{t}(x, y, \omega)=-\frac{1}{64}$.

Figure 32. Stochastic Precaustic and Prelevel Surface

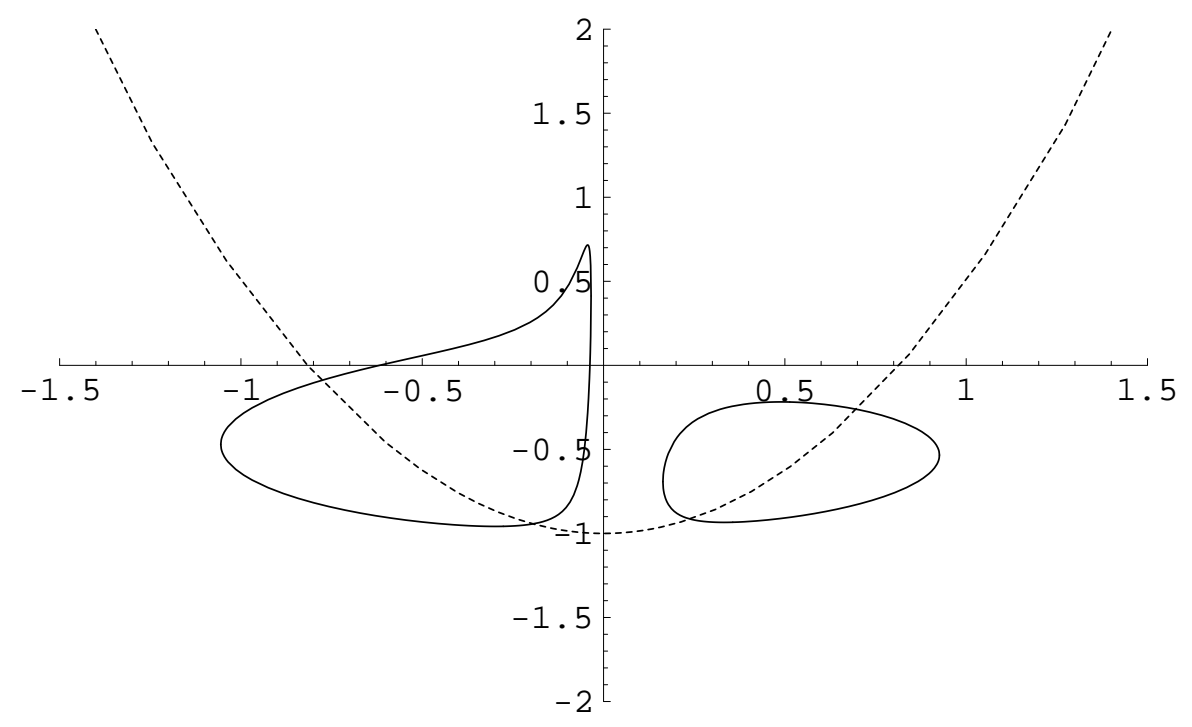

Figure 33. Stochastic Caustic and Level Surface

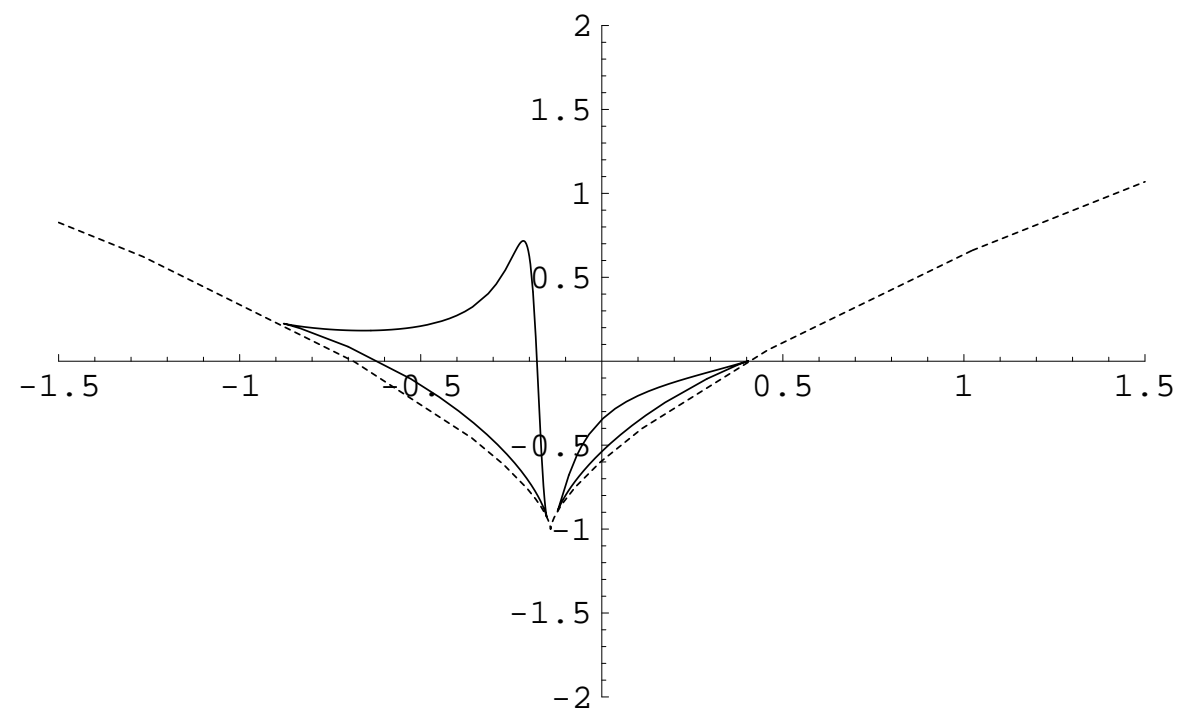




\subsection{Consequences for the Burgers Fluid}

We now recast the comments made at the end of Section 3 in the more general setting of $c$ and $k$ non-zero. We emphasise that the above results hold for any finite $\epsilon$ (the strength of the white noise) as long as $\operatorname{Det}\left(\frac{\partial^{2} \mathcal{A}}{\partial x_{0} \partial x}\right) \neq 0$. In a future paper [11] we will expound on the small $\epsilon$ case in some detail explaining why the caustics (shockwaves) are stable and the level surfaces are unstable under noisy perturbations.

Recall that our random map $\Phi_{s}(\omega): \mathbb{R}^{d} \rightarrow \mathbb{R}^{d}$ satisfies

$$
d_{s} \dot{\Phi}_{s}=-\nabla c\left(\Phi_{s}\right) d s-\epsilon \nabla k\left(\Phi_{s}, s\right) d W_{s},
$$

with $\Phi_{0}=I$ and $\dot{\Phi}_{0}=\nabla S_{0}$. The global inverse function theorem [37] gives:

Proposition 5.4. If $c, k$ and $S_{0}$ are smooth with bounded second order partial derivatives, there exists $T(\omega)>0$ such that $\Phi_{s}(\omega): \mathbb{R}^{d} \rightarrow \mathbb{R}^{d}$ is a random diffeomorphism $0 \leq s \leq T(\omega)$.

It follows that :

Proposition 5.5. The random Burgers velocity field in the limit of zero viscosity is

$$
v^{0}(x, t)=v^{0}\left(\Phi_{t}^{-1} x, 0\right)-\int_{0}^{t} \nabla c\left(\Phi_{s} \Phi_{t}^{-1} x\right) d s-\int_{0}^{t} \epsilon \nabla k\left(\Phi_{s} \Phi_{t}^{-1} x, s\right) d W_{s},
$$

with $v^{0}(x, 0)=\nabla S_{0}(x)$. This $v^{0}$ is almost surely $0 \leq t \leq T(\omega)$ a classical solution of Burgers equation. The corresponding solution of the continuity equation, the random density $\rho(x, t)=\left|\operatorname{Det}\left(\nabla \Phi_{t}^{-1} x\right)\right|$, is continuous almost surely for $0 \leq t \leq T(\omega)$ but $v^{0}$ has jump discontinuities on the random caustics. Evidently $\nabla \wedge v^{0} \equiv 0$, almost surely.

Remark 5.3. More detailed information on the behaviour of $v^{0}$ and $\rho$ on the caustic is given in Elworthy, Truman and Zhao [15].

After the caustic time $T(\omega)$, we have seen how to characterise in terms of the stochastic action when the level surface meets the caustic in cusps. In two dimensions, this enables one to divide the caustic into hot and cool parts depending on whether or not the speed of the Burgers fluid is zero on one side of the caustic. We have also seen how a zero speed condition on the precaustic and prelevel surface leads to turbulent like behaviour of the Burgers fluid at the corresponding points of the caustic. For instance the large jump discontinuity in the speed of the Burgers fluid which we see in crossing the semicubical parabolic shockwave at the cusp is caused by this 
zero speed condition. Here we should emphasise that the stochastic and deterministic behaviours are very similar if we take snapshots of the Burgers fluid fixed in time.

We now emphasise that new features emerge if one tries to use the above ideas to analyse the "intermittence" of stochastic turbulence as opposed to deterministic turbulence. We shall discuss this in detail in a future paper. The reason for the sharp contrast between stochastic and deterministic turbulence can already be seen here if we associate turbulent behaviour with a change in the number of cusped curves on the level surface. According to Proposition 5.3 and Theorem 5.2, the times $t$ when this occurs are just the times when the prelevel surfaces touch the precaustic. The times $t$ when this number of curves changes in the deterministic case are simply the zeros of a deterministic function $\zeta$, usually isolated zeros. In the stochastic case $\zeta$ is a stochastic process whose zeros usually form a perfect set i.e. an infinite set containing no isolated points. At these times the number of cusped curves changes with infinite frequency because of the infinitely rapid oscillation of the stochastic process $\zeta$. This is in line with what one would expect for turbulent behaviour. When the stochastic process $\zeta$ is recurrent this turbulent behaviour is "intermittent" so that the scale of turbulent fluctuations varies in a random periodic way. 


\section{Conclusion}

We have seen how generally the level surfaces of Hamilton's principal function meet the caustic surface in cusped curves and discussed some of the ramifications of this for Burgers turbulence. In particular the cool part of the caustic is associated with jump discontinuities in both $u^{0}$ and $v^{0}$. The results in this paper should be compared with results for Euler's equation which is identical to the above save for the constraint that the fluid density $\rho$ is unity. In a future paper we hope to relax the assumption that the Burgers velocity field is irrotational, when more realistic comparisons with Euler's equation can be made. Needless to say, the detailed nature of the geometry of the caustic surface and the level surface will affect the value of $v^{\mu}(x, t)$ on the caustic. Geometrical and analytical results are clearly relevant here for small noise in the way they relate the stochastic and deterministic situations. We will discuss some more analytical results in our future papers $[11,12]$.

The main object lesson of the present study is that the number of cusped curves on the wavefront will change infinitely rapidly in the stochastic case when the presurfaces touch and that this behaviour will recur in a random periodic way if a certain stochastic process is recurrent. This is the "intermittence" of turbulence in our model. There is no analogue of this for the deterministic Burgers equation. We shall discuss this in more detail in a future publication.

\section{Acknowledgements}

It is a pleasure to thank Sergio Albeverio, David Elworthy, Mark Freidlin, Leonid Pastur and David Williams for helpful conversations and the first two for opportunities to present these results in Bonn and Warwick, respectively.

\section{References}

[1] S. Albeverio, S. Molchanov and D. Surgailis, Stratified structure of the universe and Burgers equation - a probability approach, Probab. Theory Relat. Fields 100, 457-484 (1994)

[2] V. I. Arnol'd, Singularities of caustics and wave fronts, Mathematics and its Applications (Soviet Series), 62, (Kluwer Academic Publishers Group, Dordrecht, 1990) 
[3] V. I. Arnol'd, Mathematical Methods of Classical Mechanics, (SpringerVerlag, New York, 1989)

[4] L. Bertini, N. Cancrini and G. Jona-Lasinio, The stochastic Burgers equation, Comm. Math. Phys. 165, 211-232 (1994)

[5] Z. Brzeźniak, M. Capiński and F. Flandoli, Stochastic Navier-Stokes equations with multiplicative noise, Stochastic Anal. Appl. 10, 523 (1992)

[6] Z. Brzeźniak and S. Bolesław, On the blow-up phenomena for the NavierStokes type equations, Univ. Iagl. Acta Math. 32, 89 (1995)

[7] Z. Brzeźniak and F. Flandoli, Regularity of solutions and random evolution operator for stochastic parabolic equations, Pitman Research Notes in Mathematics Series 268, 54 (1992)

[8] G. Da Prato, A. Debusche and R. Temam, Stochastic Burgers equation, Nonlinear Differential Equations Appl 1, 389-402 (1994)

[9] I. M. Davies and A. Truman, On the Laplace asymptotic expansion of conditional Wiener Integrals and the Bender-Wu formula for $x^{2 n}$ anharmonic oscillators, J. Math. Phys. 24, 255-266 (1983)

[10] I. M. Davies and A. Truman, Laplace asymptotic expansions of conditional Wiener Integrals and generalized Mehler kernel formula for Hamiltonians on $L^{2}\left(R^{n}\right)$, J. Phys. A17, 2773 (1984)

[11] I. M. Davies, A. Truman and H. Z. Zhao, Stochastic Heat and Burgers Equations and their Singularities II - Analytical Properties, in preparation.

[12] I. M. Davies, A. Truman and H. Z. Zhao, Stochastic Heat and Burgers Equations and their Singularities III, in preparation.

[13] W. E, K. Khanin, A. Mazel and Ya Sinai, Invariant measures for Burgers equation with stochastic forcing, Ann. Math. 151, 877-960 (2000)

[14] W. E and E. Vanden Eijnden, Statistical theory for the stochastic Burgers equation in the inviscid limit, Comm. Pure Appl. Math. 53, 852-901 (2000)

[15] K. D. Elworthy, A. Truman and H. Zhao, Stochastic Elementary Formulae on Caustics I: One-Dimensional Linear Heat Equations, MRRS preprint 
[16] R. S. Ellis and J. S. Rosen, Asymptotic analysis of Gaussian integrals I, Trans. Amer. Math. Soc. 273, 447 (1982)

[17] R. S. Ellis and J. S. Rosen, Asymptotic analysis of Gaussian integrals II, Comm. Math. Phys. 82, 153 (1981)

[18] R. S. Ellis and J. S. Rosen, Laplace's Method for Gaussian integrals with an application to statistical mechanics, Ann. Probability 10, 47 (1982)

[19] M. I. Freidlin and A. D. Wentzell, Random Perturbations of Dynamical systems, (Springer-Verlag, New York, 1998)

[20] M. I. Freidlin, Probabilistic approach to the small viscosity asymptotics for the Navier-Stokes equations, Nonlinear Analysis 30, 4069 (1997)

[21] M. I. Freidlin, Functional Integration and Partial Differential Equations, (Princeton University Press, Princeton, 1985)

[22] I. Gyöngy and D. Nualart, On the stochastic Burgers' equation in the real line, Ann. Prob. 27, 782-802 (1999)

[23] H. Holden, T. Lindstrom, B. Øksendal, J. Uboe and T. S. Zhang, The Burgers' equation with a noise force and the stochastic heat equations, Comm. PDE 19, 119-141 (1994)

[24] H. Holden, T. Lindstrom, B. Øksendal, J. Uboe and T. S. Zhang, The stochastic Wick-type Burgers equation, in: Stochastic Partial Differential Equations edited by A.M. Etheridge, (London Mathematical Society Lecture Note Series 216, Cambridge University Press, 141-161, 1995)

[25] M. Kardar, G. Parisi and Y.C. Zhang, Dynamical scaling of growing interfaces, Phys, Rev. Letter 56, 889-892 (1986)

[26] Y. Kifer, The Burgers equations with a random force and a general model for directed polymers in random environments, Prob. Theory Relat. Fields 108, 29-65 (1997)

[27] H. Kunita, Stochastic Differential Equations and Stochastic Flows of Homeomorphisms, 269-291, in: Stochastic Analysis and Applications, ed. M. A. Pinsky, (Advances in Probability and Related Topics 7, Marcel Dekker, New York, 1984)

[28] R. Mikulevicius and B. L. Rozovskii, On equations of stochastic fluid mechanics, pp 285-302, Stochastics in finite and infinite dimensions, (Birkhäuser, Boston, 2001) 
[29] M. E. Taylor, Partial Differential Equations III ( Non Linear Equations), Applied Mathematical Sciences 117, (Springer-Verlag, New York, 1997)

[30] R. Tribe and O. Zaboronski, On large time asymptotics of decaying Burgers turbulence, Comm. Math. Phys. 212, 415-436 (2000)

[31] A. Truman, C. N. Reynolds, On the Swallowtail and Cusp Singularities of Burgers Equation, in preparation.

[32] A. Truman, B. T. Reynolds and C. N. Reynolds Some Exact Singularities of Burgers and Heat Equations, MRR preprint.

[33] A. Truman and H.Z. Zhao, Stochastic Burgers' equations and their semiclassical expansions, Comm. Math. Phys. 194, 231-248 (1998)

[34] A. Truman and H.Z. Zhao, The stochastic Hamilton-Jacobi equation, stochastic heat equation and Schrödinger equations, pp 441-464, Stochastic Analysis and Applications, (World Scientific, River Edge, 1996)

[35] A. Truman and H.Z. Zhao, Stochastic Hamilton-Jacobi equations and related topics, LMS Lecture Note Series 216, 287-303 (1995)

[36] A. Truman and H.Z. Zhao, On stochastic diffusion equations and stochastic Burgers' equations, J. Math. Phys. 37, 283-307 (1996)

[37] A. Truman and H.Z. Zhao, Quantum Mechanics of charged particles in random electromagnetic fields, J. Math. Phys. 37, 3180-3197 (1996)

[38] S. F. Shandarin and Ya. B Zeldovich, The large-scale structure of the universe: turbulence, intermittency, structures in a self gravitating medium, Rev. Mod. Phys. 6, 185-220 (1989) 